\title{
EXACT BOUNDARY CONTROLLABILITY OF 3-D EULER EQUATION
}

\author{
OLIVIER GLASS ${ }^{1}$
}

\begin{abstract}
We prove the exact boundary controllability of the 3-D Euler equation of incompressible inviscid fluids on a regular connected bounded open set when the control operates on an open part of the boundary that meets any of the connected components of the boundary.

Résumé. Nous prouvons la contrôlabilité exacte frontière de l'équation d'Euler des fluides parfaits incompressibles tridimensionnels dans un domaine borné et régulier, lorsque le contrôle opère sur une partie ouverte du bord qui en rencontre toutes les composantes connexes.
\end{abstract}

AMS Subject Classification. 93B05, 35Q30, 76C99, 93C20.

Received December 16, 1998. Revised March 29, 1999.

\section{INTRODUCTION}

Let $\Omega$ be a non-empty, open, connected, bounded and regular (say $C^{\infty}$-regular) subset of $\mathbb{R}^{3}$. Let $\Gamma_{0}$ be an open and non-empty subset of its boundary $\partial \Omega$, which meets any connected component of $\partial \Omega$. We are interested in the exact boundary controllability of the 3-D Euler equation of inviscid incompressible fluids for $\left(\Omega, \Gamma_{0}\right)$, that is, the following question: given $T>0$, given $y_{0}$ and $y_{1}$ two solenoidal vector fields, i.e. satisfying

$$
\operatorname{div} y_{0}=\operatorname{div} y_{1}=0 \text { in } \Omega,
$$

regular (in this paper, $C^{2, \alpha}$ for some Hölder coefficient $\left.\alpha \in(0,1)\right)$ and which satisfy

$$
y_{0} . n=y_{1} . n=0 \text { on } \partial \Omega \backslash \Gamma_{0},
$$

where $n$ is the outward unit normal vector field on $\partial \Omega$, does there exist a solution $y$ of the Euler system

$$
\partial_{t} y+(y \cdot \nabla) y=\nabla p \text { in } \Omega \times[0, T]
$$

for some $p \in \mathfrak{D}^{\prime}(\Omega \times(0, T))$ and

$$
\operatorname{div} y=0 \text { in } \Omega \times[0, T],
$$

Keywords and phrases: Controllability, boundary control, Euler equation for ideal incompressible fluids.

${ }^{1}$ Université Paris-Sud, Analyse numérique et EDP, 91405 Orsay, France; e-mail: Olivier.Glass@math.u-psud.fr 
with

$$
y(x, t) . n(x)=0, \quad \forall t \in[0, T], \forall x \in \partial \Omega \backslash \Gamma_{0},
$$

and such that

$$
\begin{gathered}
y_{\mid t=0}=y_{0} \text { in } \Omega, \\
y_{\mid t=T}=y_{1} \text { in } \Omega ?
\end{gathered}
$$

This problem, raised by Lions in [10], was solved by Coron in [3] and [4] in the two-dimensional case. In a previous paper [6], we have sketched a proof of a solution to this problem in dimension 3 when $\Omega$ is simply connected. Here we give the details of the demonstration and prove that, as announced in [7], the result still holds when $\Omega$ is not necessarily simply connected. Actually, we prove the following result:

Theorem 1.1. Given $\alpha \in(0,1)$, two functions $y_{0}$ and $y_{1}$ in $C^{2, \alpha}\left(\bar{\Omega} ; \mathbb{R}^{3}\right)$ satisfying (1.1) and (1.2) and $T>0$, then there exists a function $y$ in the space $C\left([0, T] ; C^{1, \alpha}\left(\bar{\Omega} ; \mathbb{R}^{3}\right)\right) \cap L^{\infty}\left([0, T] ; C^{2, \alpha}\left(\bar{\Omega} ; \mathbb{R}^{3}\right)\right)$ such that (1.3) to (1.7) hold for some $p \in \mathfrak{D}^{\prime}(\Omega \times(0, T))$.

Remark 1.2. As noticed in [4], the condition that $\Gamma_{0}$ meets any connected component of the boundary is necessary for the exact controllability as a consequence of the Kelvin law.

Indeed, suppose that we choose $y_{1}=0$ on some connected component $\Gamma^{*}$ of the boundary, which does not meet $\Gamma_{0}$. Then the existence of $y$ and the Kelvin law for any loop $\gamma$ in this connected component of the boundary imply that

$$
\int_{\gamma} y_{0} d \tau=\int_{\tilde{\gamma}} y_{1} d \tau=0
$$

where $\tilde{\gamma}$ is the loop obtained when transporting $\gamma$ by the flow of $y$. This necessarily implies that $y_{0 \mid \Gamma^{*}}$ is a gradient, which is not generally the case.

Now we briefly describe the method. As in [3] and [4], the steps of the proof of Theorem 1.1 are the following: first, we prove that this question can be reduced to the problem of zero-controllability with small initial data (that is $y_{1}=0$ and $\left.\left\|y_{0}\right\|_{C^{2, \alpha}\left(\bar{\Omega} ; \mathbb{R}^{3}\right)}<\epsilon\right)$ and small time $T$.

To be more precise, we prove in section 7 that Theorem 1.1 is a consequence of the following proposition:

Proposition 1.3. There exists $\nu>0$ such that if $y_{0} \in C^{2, \alpha}\left(\bar{\Omega} ; \mathbb{R}^{3}\right)$ satisfies (1.1), (1.2) and $\left\|y_{0}\right\|_{C^{2, \alpha}\left(\bar{\Omega}, \mathbb{R}^{3}\right)}<\nu$, then there exists a function $y$ in the space $C\left([0,1] ; C^{1, \alpha}\left(\bar{\Omega} ; \mathbb{R}^{3}\right)\right) \cap L^{\infty}\left([0,1] ; C^{2, \alpha}\left(\bar{\Omega} ; \mathbb{R}^{3}\right)\right)$ and $p \in \mathfrak{D}^{\prime}(\Omega \times(0,1))$ satisfying (1.3) to (1.7) for $y_{1}=0$, and $T=1$, and also

$$
y=0 \text { and } p=0, \quad \forall t \in\left[\frac{1}{2}, 1\right] .
$$

In order to prove the last proposition, we use a method called the "return method", used in [3] and [4] and introduced in [2] for a stabilization problem. Precisely, - since the linearized Euler equation around $y \equiv 0$ is not controllable - we consider the linearized system around other solutions of the Euler control system $\bar{y}$ satisfying $\bar{y}_{\mid t=0}=\bar{y}_{\mid t=1}=0$ (a kind of "loop"). If this linearized control system is controllable, then for $y_{0}$ small enough, one can hope to find $y$ close to $\bar{y}$ answering to the general problem. In order to prove the existence of $y$, we use a construction of solutions of the Euler system due to Bardos and Frisch (see [1]).

In the previous presentation of the problem, the control itself was not explicit. As a control, we can take for example $y . n$ on $\Gamma_{0} \times[0, T]$ and the tangent part of the vorticity where the fluid enters, that is $\omega \wedge n$ where $y . n<0$ in $\Gamma_{0}$ (see for that $[9]$ ). 
In the next section, we will present the different tools we need to introduce the particular solution $\bar{y}$. This function will be found in the particular potential form " $\nabla \theta$ ", in order for its flow to satisfy precise properties. In the simply connected case, as in dimension $2, \bar{y}$ has the property that any particle in $\bar{\Omega}$ following the flow of $\bar{y}$ must go out of $\bar{\Omega}$. The major difference is that in dimension 3, as in the 2-D case for the Navier-Stokes equation [5], this " $\nabla \theta$ " can no longer be chosen stationary. In the multi-connected case, we will have to introduce an other type of " $\nabla \theta$ " (which we need to append to the previous one), whose flow moves certain Jordan curves properly.

In Section 3, we define a function $F$ on a certain functional set, of which $y$ will be found as a fixed point. Given $y$ near $\bar{y}, F$ associates the solution of a linear control problem relied to (1.3-1.7).

In Section 4, we prove Proposition 1.3, by showing that $F$ admits a fixed point which gives a solution to the non-linear problem.

Section 5 deduces Theorem 1.1 from Proposition 1.3.

Section 6 is devoted to the proof of Lemma 2.1, which corresponds to the first type of " $\nabla \theta$ ".

Section 7 corresponds to the second type of " $\nabla \theta$ " presented in Lemma 2.3.

In Sections 8 and 9, we give the details of the proofs of technical lemmas needed in Sections 5 and 6 respectively.

\section{The particular solution of Euler system: $\bar{y}$} $\bar{\Omega}$.

We first set up the following lemma, which stands for any regular bounded open set $\tilde{\Omega}$ such that $\tilde{\Omega}$ contains

Lemma 2.1. For all a in $\bar{\Omega}$, there exists $\theta \in C^{\infty}(\overline{\widetilde{\Omega}} \times[0,1] ; \mathbb{R})$ satisfying:

$$
\begin{gathered}
\text { Supp } \theta \subset \tilde{\Omega} \times(0,1), \\
\theta=0 \text { in } \bar{\Omega} \times\left(\left[0, \frac{1}{4}\right] \cup\left[\frac{3}{4}, 1\right]\right), \\
\Delta \theta=0 \text { in } \bar{\Omega} \times[0,1], \\
\frac{\partial \theta}{\partial n}=0 \text { on }\left(\partial \Omega \backslash \Gamma_{0}\right) \times[0,1], \\
\phi^{\nabla \theta}(a, 0,1) \in \tilde{\Omega} \backslash \bar{\Omega},
\end{gathered}
$$

where we denote by $\phi^{\nabla \theta}: \overline{\tilde{\Omega}} \times[0,1] \times[0,1] \longrightarrow \overline{\tilde{\Omega}},\left(x, t_{1}, t_{2}\right) \mapsto \phi^{\nabla \theta}\left(x, t_{1}, t_{2}\right)$ the flow of $\nabla \theta$, i.e. the function which satisfies

$$
\begin{array}{r}
\frac{\partial \phi}{\partial t_{2}}=\nabla \theta\left(\phi, t_{2}\right), \\
\phi\left(x, t_{1}, t_{1}\right)=x .
\end{array}
$$

With the help of that lemma, we will be able to single out a solution of the Euler system, which makes each part of the fluid go out $\bar{\Omega}$ (far enough), and then go back the same way.

In the multi-connected case, we will also need another type of " $\nabla \theta$ ", in order to control irrotational flows which class in de Rham's cohomology first space is not trivial. Let us describe these flows (we refer to [11], Appendix I).

We introduce, in the multi-connected case, precisely when $H_{1}(\Omega)=\mathbb{Z}^{s}$ with $s \geq 1, s$ smooth hypersurfaces $\Sigma_{1}, \ldots, \Sigma_{s}$ (see for example [11]) included in $\bar{\Omega}$ and with boundaries in $\partial \Omega$, the intersection being transverse, 
and which neutral intersections (if needed) are also transverse.

$$
\Omega \backslash\left(\bigcup_{i=1}^{s} \Sigma_{i}\right) \text { is simply connected. }
$$

For $i \in\{1, \ldots, s\}$, we distinguish the two sides $\Sigma_{i}$, that we denote by $\Sigma_{i}^{+}$and $\Sigma_{i}^{-}$. For a function $f$ defined in $\Omega \backslash \Sigma_{i}$, which trace on $\Sigma_{i}^{+}$may differ from the one on $\Sigma_{i}^{-}$, one defines $[f]_{i}:=f_{\mid \Sigma_{i}^{+}}-f_{\mid \Sigma_{i}^{-}}$considered as a function on $\Sigma_{i}$.

Then using the Lax-Milgram theorem on the functional space:

$$
X_{i}:=\left\{p \in H^{1}\left(\Omega \backslash \bigcup_{i=1}^{s} \Sigma_{i}\right) /[p]_{i}=\text { constant, }\left[p_{j}\right]=0 \text { for } j \neq i\right\}
$$

one easily deduces the existence of a function $q_{i}^{\prime}$ in $X_{i}$ such that:

$$
\int_{\Omega} \nabla q_{i}^{\prime} \cdot \nabla p=[p]_{i}, \forall p \in X_{i}
$$

This leads to the existence of a function $q_{i} \in X_{i}$ such that:

$$
\begin{gathered}
\Delta q_{i}=0 \text { in } \Omega \backslash \Sigma_{i}, \\
\partial_{n} q_{i}=0 \text { on } \partial \Omega, \\
{\left[q_{i}\right]_{i}=1,} \\
{\left[q_{i}\right]_{j}=0 \text { for } j \neq i,} \\
{\left[\partial_{n} q_{i}\right]_{i}=0 .}
\end{gathered}
$$

By $(2.11,2.12)$ and $(2.13)$, the $\mathcal{Q}_{i}:=\nabla q_{i}$ are in $C^{0}(\Omega)$ and, in fact in $C^{\infty}(\bar{\Omega})$ (see [11], Appendix I, Rem. 1.3.ii).

Remark 2.2. As it is known (see again [11]), any (regular) vector field $X$ satisfying

$$
\text { curl } X=0 \text {, }
$$

can then be written as

$$
X=\nabla \chi+\sum_{i=1}^{s} \alpha_{i} \mathcal{Q}_{i}
$$

for some $\chi$ and $\alpha_{i}, i \in\{1, \ldots, s\}$. If we add to (2.14) the conditions:

$$
\operatorname{div} X=0 \text { in } \Omega, X . n=0 \text { on } \partial \Omega \text {, }
$$

then the previous $\chi$ is zero, and we describe only the first cohomology space.

Let us emphasize that $\operatorname{curl} X=0, \operatorname{div} X=0$ in $\Omega$ and $X . n=0$ on $\partial \Omega$ does not imply that $X=0$. Indeed, for all $i \in\{1, \ldots, s\}$, we have $\operatorname{curl} \mathcal{Q}_{i}=0, \operatorname{div} \mathcal{Q}_{i}=0$ in $\Omega$ and $\mathcal{Q}_{i} \cdot n=0$ on $\partial \Omega$, but $\mathcal{Q}_{i} \neq 0$. This fact will oblige us to set up a second lemma to define our particular solution $\bar{y}$ and to get rid of the terms " $\mathcal{Q}_{i}$ ". It is in particular necessary to treat the problem with (for instance) $y_{0}=\mathcal{Q}_{i}$ and $y_{1}=0$. Roughly speaking, the following lemma solves this precise case. 
Lemma 2.3. There exists $\bar{\nu}>0$, such that for $i$ in $\{1, \ldots, s\}$, there exists $\theta^{i} \in C^{\infty}(\bar{\Omega} \times[0,1] ; \mathbb{R})$ and $\aleph^{i} \in$ $C^{\infty}\left(\bar{\Omega} ; \mathbb{R}^{3}\right)$ satisfying:

$$
\begin{gathered}
\text { Supp } \theta^{i} \subset \tilde{\Omega} \times(0,1), \\
\theta^{i}=0 \text { in } \bar{\Omega} \times\left(\left[0, \frac{1}{4}\right] \cup\left[\frac{3}{4}, 1\right]\right), \\
\Delta \theta^{i}=0 \text { in } \bar{\Omega} \times[0,1], \\
\frac{\partial \theta^{i}}{\partial n}=0 \text { on } \partial \Omega \backslash \Gamma_{0} \times[0,1], \\
\text { Supp } \aleph^{i} \subset \tilde{\Omega} \backslash \bar{\Omega},
\end{gathered}
$$

and such that for any $f \in C\left([0,1], C^{2, \alpha}\left(\overline{\widetilde{\Omega}} ; \mathbb{R}^{3}\right)\right)$ with

$$
\left\|f-\nabla \theta^{i}\right\|_{C([0,1] \times \bar{\Omega})}<\bar{\nu}
$$

if we define $w^{i} \in C^{\infty}\left(\overline{\widetilde{\Omega}} \times[0,1] ; \mathbb{R}^{3}\right)$ by

$$
\begin{gathered}
w^{i}(\cdot, 0)=\operatorname{curl}\left(\aleph^{i}\right) \text { on } \overline{\tilde{\Omega}} \\
\partial_{t} w^{i}+(f . \nabla) w^{i}=\left(w^{i} \cdot \nabla\right) f-w^{i} \operatorname{div} f \text { on } \tilde{\Omega} \times[0,1],
\end{gathered}
$$

and if we define the function $\zeta^{i}$ in $C^{\infty}\left(\overline{\widetilde{\Omega}} \times[0,1] ; \mathbb{R}^{3}\right)$ by

$$
\begin{gathered}
\operatorname{curl} \zeta^{i}=w^{i} \text { in } \bar{\Omega} \times[0,1], \\
\operatorname{div} \zeta^{i}=0 \text { in } \bar{\Omega} \times[0,1], \\
\zeta^{i} \cdot n=\partial_{n} \theta^{i} \text { on } \partial \Omega \times[0,1], \\
\int_{\Omega} \zeta^{i}(0) \cdot \mathcal{Q}_{j} d x=0, \\
\int_{\Omega}\left(\partial_{t} \zeta^{i}+f \wedge \operatorname{curl}\left(\zeta^{i}\right)\right) \cdot \mathcal{Q}_{j} d x=0, \quad \forall j \in\{1, \ldots, s\},
\end{gathered}
$$

then we have

$$
\operatorname{Supp} w^{i}(\cdot, 1) \subset \tilde{\Omega} \backslash \bar{\Omega}
$$

and

$$
\zeta^{i}(1)=\mathcal{Q}_{i}
$$

As we will see in Section $6, \bar{y}:=\nabla \theta$ in this lemma will be chosen, not in terms of the flow of points, but in terms of the flow of certain Jordan curves.

We can now present what our particular solution to Euler system $\bar{y}$ will be.

We denote by $B\left(x_{i}, r_{i}\right)$ the open ball of center $x_{i}$ and of radius $r_{i}$, and by $\bar{B}\left(x_{i}, r_{i}\right)$ its closure. By Lemma 2.1, one can find by compactness of $\bar{\Omega}$ a positive integer $k, k$ points $x_{i}$ in $\bar{\Omega}, k$ real numbers $r_{i}>0$ and $k$ smooth 
functions $\theta_{i} \in C^{\infty}(\bar{\Omega} \times[0,1], \mathbb{R}), i \in\{1, \ldots, k\}$, satisfying $(2.1-2.4)$, and an open bounded regular set $\Omega_{2}$ with $\bar{\Omega} \subset \Omega_{2}$ and also $\overline{\Omega_{2}}$ such that

$$
\begin{gathered}
\bar{B}\left(x_{i}, r_{i}\right) \subset \tilde{\Omega}, \\
\bar{\Omega} \subset \bigcup_{i=1}^{i=k} B\left(x_{i}, r_{i}\right), \\
\phi^{\nabla \theta_{i}}\left(\bar{B}\left(x_{i}, r_{i}\right), 0,1\right) \cap \bar{\Omega}_{2}=\emptyset .
\end{gathered}
$$

Let us split the time-segments $[1 / 4,1 / 2]$ and $[1 / 2,3 / 4]$ as follows:

$$
\begin{gathered}
t_{i}=\frac{1}{4}+i \frac{1}{4 k}, \quad \forall i \in\{0, \ldots, k\}, \\
t_{i+\frac{1}{2}}=\frac{1}{4}+\left(i+\frac{1}{2}\right) \frac{1}{4 k}, \quad \forall i \in\{0, \ldots, k-1\}, \\
t_{i}=\frac{1}{2}+(i-k) \frac{1}{4 s}, \quad \forall i \in\{k, \ldots, k+s\} .
\end{gathered}
$$

We can now define $\theta$ in $C^{\infty}(\tilde{\Omega} \times[0,1 / 2], \mathbb{R})$ by

$$
\begin{gathered}
\theta(x, t)=0, \forall(x, t) \in \bar{\Omega} \times\left[0, \frac{1}{4}\right], \\
\theta(x, t)=8 k \theta_{j}\left(x, 8 k\left(t-t_{j-1}\right)\right), \quad \forall j \in\{1, \ldots, k\}, \quad \text { and } \forall(x, t) \in \tilde{\Omega} \times\left[t_{j-1}, t_{j-\frac{1}{2}}\right], \\
\theta(x, t)=-8 k \theta_{j}\left(x, 8 k\left(t_{j}-t\right)\right), \quad \forall j \in\{1, \ldots, k\}, \quad \text { and } \forall(x, t) \in \tilde{\Omega} \times\left[t_{j-\frac{1}{2}}, t_{j}\right] .
\end{gathered}
$$

During the interval of time $\left[\frac{1}{2}, 1\right]$, we define $\theta$ by

$$
\begin{array}{r}
\theta(x, t)=4 s \theta^{j-k+1}\left(x, 4 s\left(t-t_{j}\right)\right), \quad \forall j \in\{k, \ldots, k+s-1\}, \\
\text { and } \forall(x, t) \in \tilde{\Omega} \times\left[t_{j}, t_{j+1}\right] .
\end{array}
$$

Let $\bar{y}:=\nabla \theta$. We remark that $\bar{y}$ restricted to $\bar{\Omega} \times[0,1]$ is a $C^{\infty}$ solution of $(1.3-1.7)$ with $T=1, y_{0}=y_{1}=0$ and with $p(x, t)=\partial \theta / \partial t+|\nabla \theta|^{2} / 2$.

\section{The Application $F$}

\subsection{Introduction}

In this section,we use this particular solution to single out the application $F$, the fixed point of which gives a solution for Proposition 1.3. For that purpose we first introduce a certain functional set $X_{\nu}$. 
Let $\mu:[0,1] \rightarrow[0,1] C^{\infty}$-regular, such that

$$
\left\{\begin{array}{l}
0 \leq \mu \leq 1 \text { in }[0,1], \\
\mu=1 \text { in }[0,1 / 8], \\
\mu=0 \text { in }[1 / 4,1] .
\end{array}\right.
$$

Then the set $X_{\nu}$ for $\nu>0$ small enough, is defined as:

$$
\begin{aligned}
& X_{\nu}=\left\{u \in C^{0}\left([0,1], C^{2, \alpha}\left(\bar{\Omega} ; \mathbb{R}^{3}\right)\right) / \operatorname{div} u=0, \text { in } \Omega\|u-\bar{y}\|_{C^{0}(\bar{\Omega} \times[0,1])}<\nu,\right. \\
&\left.u(x, t) \cdot n(x)=\mu(t) y_{0}(x) \cdot n(x)+\bar{y} \cdot n \text { on } \partial \Omega \times[0,1]\right\} .
\end{aligned}
$$

The value of $F$ will be a solution to a certain linear controllability problem in $C\left([0,1], C^{2, \alpha}(\bar{\Omega})\right)$.

We introduce a linear operator $\pi$ which extends functions defined on $\bar{\Omega}$ to functions defined on $\bar{\Omega}$, and with support in $\Omega_{2}$. We will require also for it to send continuously $C^{[\lambda], \lambda-[\lambda]}\left(\bar{\Omega} ; \mathbb{R}^{3}\right)$ into $C^{[\lambda], \lambda-[\lambda]}\left(\bar{\Omega} ; \mathbb{R}^{3}\right)$, for all $\lambda \in[0,3) \backslash \mathbb{N}$.

Now we define the application $F$. For $u \in X_{\nu}$, we set

$$
\tilde{u}=\bar{y}+\pi(u-\bar{y}) .
$$

Then $F(u)$ will be a solution of the following problem:

$$
\begin{gathered}
F(u)(\cdot, 0)=y_{0} \text { in } \bar{\Omega}, \\
F(u)(\cdot, \cdot) . n=0 \text { on }[0,1] \times\left(\partial \Omega \backslash \Gamma_{0}\right), \\
\operatorname{div} F(u)=0 \text { in }[0,1] \times \Omega,
\end{gathered}
$$

and if we set $\omega:=\operatorname{curl}(F(u))$, then it should satisfy

$$
\begin{gathered}
\int_{\Omega}\left(\partial_{t} F(u)+\tilde{u} \wedge \omega\right) \cdot \mathcal{Q}_{i}=0 \text { in }[0,1], \forall i \in\{1, \ldots, s\}, \\
\partial_{t} \omega+(\tilde{u} . \nabla) \omega=(\omega . \nabla) \tilde{u} \text { in }[0,1] \times \Omega .
\end{gathered}
$$

The controllability problem is to find a $F(u)$ such that

$$
F(u)(1, \cdot)=0 \text { in } \bar{\Omega} .
$$

Of course, this linear problem becomes "close" to the Euler problem as $\omega$ approaches curl $u$.

\subsection{Preliminaries}

Before making $F$ explicit, we introduce some notations.

For a regular open bounded subset $E$ of $\mathbb{R}^{3}$, we denote by $\|\cdot\|_{i, \alpha, E}$ for $i \in \mathbb{N}$ and $\alpha \in(0,1)$, the usual norm for $C^{i, \alpha}(\bar{E})$ and by $\|\cdot\|_{i, E}$ the usual norm for $C^{i}(\bar{E})$. 
We introduce a partition of unity adapted to the open covering of $\bar{\Omega}$ by the open sets $B\left(x_{i}, r_{i}\right)$ (described in $(2.30-2.32))$, that is some functions $\kappa_{i} \in C_{0}^{\infty}(\tilde{\Omega} ;[0,1])$ such that

$$
\begin{array}{r}
\text { Supp } \kappa_{i} \subset B\left(x_{i}, r_{i}\right), \\
\sum_{i=1}^{i=k} \kappa_{i} \equiv 1 \text { in } \bar{\Omega} .
\end{array}
$$

In this section, we will frequently use the following lemma, of which we postpone the demonstration to Section 3.4.

Lemma 3.1. Let $U$ be a function in $C^{0}\left([0, T], C^{2, \alpha}\left(\overline{\tilde{\Omega}}, \mathbb{R}^{3}\right)\right)$, and $W_{0}$ be a function in $C^{1, \alpha}\left(\overline{\widetilde{\Omega}}, \mathbb{R}^{3}\right)$. Let $W$ be a function in $C^{0}\left([0, T], C^{1, \alpha}\left(\overline{\widetilde{\Omega}}, \mathbb{R}^{3}\right)\right)$ defined by the following system

$$
\left\{\begin{array}{l}
W(\cdot, 0)=W_{0} \text { in } \overline{\tilde{\Omega}} \\
\partial_{t} W+(U . \nabla) W=(W . \nabla) U-(\operatorname{div} U) W \text { in } \tilde{\Omega} \times[0, T] .
\end{array}\right.
$$

Then for all $t \in[0, T]$, one has

$$
\operatorname{div} W(\cdot, t)=0
$$

Moreover, if $\operatorname{div} U=0$ in $\Omega$ and $W_{0}=$ curl $V_{0}$ in $\Omega$, then there exists $V \in C^{0}\left([0, T], C^{2, \alpha}\left(\overline{\tilde{\Omega}}, \mathbb{R}^{3}\right)\right)$ such that for all $t \in[0, T]$

$$
W(t)=\operatorname{curl} V(t) \text { in } \Omega
$$

\subsection{Construction of $F$}

We now give an explicit formulation of $F(u)$. Let $u \in X_{\nu}$ for $\nu$ small enough ( $\operatorname{say} \nu<\nu_{0}$ with $\nu_{0}<\bar{\nu}$ ). We associate $\tilde{u}$ defined by (3.3).

We define $F(u)$ by its curl $\omega$ in $\Omega$ and by "coordinate" $\lambda_{i}$ with respect to the functions $\mathcal{Q}_{i}$. We define the functions $\omega$ and $\lambda_{i}$ in a first step, during the times [0,1/2], and then we define them in the interval [1/2,1].

Along the construction of $F$, we will allow ourselves to reduce $\nu_{0}$ in order to make $F$ correctly defined.

We introduce a first function $\omega^{*}$ in $C^{0}\left([0,1], C^{1, \alpha}\left(\overline{\tilde{\Omega}} ; \mathbb{R}^{3}\right)\right)$. We define $\omega^{*}$ by the relations

$$
\left\{\begin{array}{l}
\omega^{*}(\cdot, 0)=\operatorname{curl}\left(\sum_{i=1}^{k}\left(\kappa_{i} \pi\left(y_{0}\right)\right)\right) \text { in } \bar{\Omega} \\
\partial_{t} \omega^{*}+(\tilde{u} \cdot \nabla) \omega^{*}=\left(\omega^{*} \cdot \nabla\right) \tilde{u}-(\operatorname{div} \tilde{u}) \omega^{*} \text { in } \tilde{\Omega} \times(0,1) .
\end{array}\right.
$$

By Lemma $3.1, \omega^{*}(\cdot, 1 / 4)$ is a curl in $\Omega$ : let us say

$$
\omega^{*}(\cdot, 1 / 4)=\operatorname{curl} \mathcal{W} \text { in } \Omega
$$

with $\mathcal{W} \in C^{2, \alpha}(\overline{\tilde{\Omega}})$.

We define then the functions $w^{l}$ in $C^{0}\left([1 / 4,1 / 2], C^{1, \alpha}\left(\overline{\tilde{\Omega}} ; \mathbb{R}^{3}\right)\right)$ by the equations:

$$
\left\{\begin{array}{l}
w^{l}(\cdot, 1 / 4)=\operatorname{curl}\left(\kappa_{l} \pi(\mathcal{W})\right) \text { in } \overline{\tilde{\Omega}} \\
\partial_{t} w^{l}+(\tilde{u} \cdot \nabla) w^{l}=\left(w^{l} \cdot \nabla\right) \tilde{u}-(\operatorname{div} \tilde{u}) w^{l} \text { in } \tilde{\Omega} \times[1 / 4,1 / 2]
\end{array}\right.
$$


Of course, we have the relation for $t \in[1 / 4,1 / 2]$

$$
\omega^{*}=\sum_{l=1}^{l=k} w^{l}
$$

Let us now build $\omega: \bar{\Omega} \times[0,1] \longrightarrow \mathbb{R}^{3}$, continuous in the variable $t$ from $[0,1] \backslash\left\{t_{i-\frac{1}{2}}, i \in\{1, \ldots, k\}\right\}$ into $C^{1, \alpha}\left(\bar{\Omega} ; \mathbb{R}^{3}\right)$, continuous at the right of each $t_{i-\frac{1}{2}}$ and with a limit in $C^{1, \alpha}\left(\overline{\widetilde{\Omega}} ; \mathbb{R}^{3}\right)$ at the left of each $t_{i-\frac{1}{2}}$ (for $i \in\{1, \ldots, k\})$. We will extract $F(u)$ from this $\omega$.

Let us define $\omega$ this way :

$$
\omega(x, t)=\omega^{*}(x, t) \text { in } \overline{\tilde{\Omega}} \times\left[0, \frac{1}{4}\right],
$$

then for $t \in[1 / 4,1 / 2]$ :

$\partial_{t} \omega+(\tilde{u} . \nabla) \omega=(\omega \cdot \nabla) \tilde{u}-(\operatorname{div} \tilde{u}) \omega, \quad$ in $\left\{\left[\frac{1}{4}, t_{\frac{1}{2}}\right] \bigcup_{i=1}^{i=k-1}\left(t_{i-\frac{1}{2}}, t_{i+\frac{1}{2}}\right) \cup\left[t_{k-\frac{1}{2}}, \frac{1}{2}\right]\right\} \times \tilde{\Omega}$.

Thus to define $\omega$ properly, we have yet to define it at times $t_{i-\frac{1}{2}}$. We do it in order that at time $t_{i}$ only $\sum_{l=i+1}^{k} w^{l}\left(x, t_{i}\right)$ stays on $\Omega$, instead of $\omega^{*}\left(x, t_{i}\right)$. For that, we simply have to consider $\omega$ at time $t_{i-\frac{1}{2}}^{-}$. Let us suppose by induction that, one has

$$
\omega\left(\cdot, t_{i-\frac{1}{2}}^{-}\right)=\sum_{l=i}^{k} w^{l}\left(\cdot, t_{i-\frac{1}{2}}\right) .
$$

Relations (2.32) and (3.3) imply that for $\|u-\bar{y}\|_{C^{0}(\bar{\Omega} \times[0,1])}$ small enough (that is, for a suitable choice of $\left.\nu_{0}\right)$, one has

$$
\phi^{\tilde{u}}\left(\bar{B}\left(x_{i}, r_{i}\right), 0, t_{i-\frac{1}{2}}\right) \cap \bar{\Omega}=\emptyset .
$$

But by (3.10) and (3.17), at time 0 , the support of $w^{i}$ is included in $\bar{B}\left(x_{i}, r_{i}\right)$. It follows from the form (3.17) that the support of $w^{i}$ follows the flow of $\tilde{u}$. We deduce that

$$
\text { Supp } w^{i}\left(t_{i-\frac{1}{2}}, \cdot\right) \cap \bar{\Omega}=\emptyset .
$$

Then, we just have to define

$$
\omega\left(x, t_{i-\frac{1}{2}}^{+}\right)=\sum_{l=i+1}^{k} w^{l}\left(\cdot, t_{i-\frac{1}{2}}\right)
$$

with the convention

$$
\omega\left(x, t_{k-\frac{1}{2}}^{+}\right)=0 .
$$

So (3.20) and (3.22) do completely define $\omega$ for $t$ in $[1 / 4,1 / 2]$. Note that by $(3.17,3.20)$ and $(3.22)$, one gets

$$
\omega(\cdot, t)=\sum_{l=i+1}^{k} w^{l}(\cdot, t), \forall t \in\left[t_{i-\frac{1}{2}}, t_{i+\frac{1}{2}}\right] .
$$


This way, we get that the restriction of $\omega$ to $\bar{\Omega} \times\left[0, \frac{1}{2}\right]$ is $C\left(\left[0, \frac{1}{2}\right], C^{1, \alpha}(\bar{\Omega})\right)$-regular and that we have in $\Omega \times\left[0, \frac{1}{2}\right]$ the relation

$$
\partial_{t} \omega+(\tilde{u} . \nabla) \omega=(\omega \cdot \nabla) \tilde{u}
$$

Furthermore, by Lemma 3.1, $\omega$ stays divergence-free in $\tilde{\Omega} \times\left[0, \frac{1}{2}\right]$.

We want to define $v$ in $C\left(\left[0, \frac{1}{2}\right], C^{1, \alpha}\left(\bar{\Omega} ; \mathbb{R}^{3}\right)\right)$ by

$$
\begin{gathered}
\operatorname{curl} v=\omega \text { in } \Omega \times\left[0, \frac{1}{2}\right], \\
\operatorname{div} v=0 \text { in } \Omega \times\left[0, \frac{1}{2}\right], \\
v \cdot n=\mu(t) y_{0} \cdot n+\bar{y} \cdot n \text { in } \partial \Omega \times\left[0, \frac{1}{2}\right], \\
\int_{\Omega} v \cdot \mathcal{Q}_{i} d x=0, \forall t \in\left[0, \frac{1}{2}\right] .
\end{gathered}
$$

But to prove that it is possible, let us point out that, for the existence of such a $v$, we need, in addition to $\operatorname{div} \omega=0$, the fact that $\omega$ is a curl in $\Omega$. This is proved also by Lemma 3.1.

By the way, we remark that the relation (3.29) is necessary to obtain the unicity of $v$.

Now, we can see that any

$$
v^{\prime}:=v+\sum_{j=1}^{s} \lambda_{j}(t) \mathcal{Q}_{j}(x)
$$

still satisfies $(3.26,3.27)$ and $(3.28)$, for any choice of $\lambda_{i}$. We choose $\lambda_{i}$, and hence $v^{\prime}$ such that

$$
\begin{gathered}
\int_{\Omega} v^{\prime}(0) \cdot \mathcal{Q}_{i} d x=\int_{\Omega} y_{0} \cdot \mathcal{Q}_{i} d x \\
\int_{\Omega}\left(\partial_{t} v^{\prime}+\tilde{u} \wedge \omega\right) \cdot \mathcal{Q}_{i} d x=0, \quad \forall i \in\{1, \ldots, s\}, \forall t \in\left[0, \frac{1}{2}\right] .
\end{gathered}
$$

Note that this is made possible because the matrix

$$
\left(\int_{\Omega} \mathcal{Q}_{i} \cdot \mathcal{Q}_{j} d x\right)_{1 \leq i \leq s, 1 \leq j \leq s},
$$

is invertible, as the $\left(\mathcal{Q}_{i}\right)$ is a free family.

We are now able to define $\omega$ in the time-interval $\left(\frac{1}{2}, \frac{3}{4}\right)$. We consider $i \in\{k, \ldots, k+s-1\}$. Let $\tilde{w}^{i} \in$ $C\left(\left[t_{i}, t_{i+1}\right], C^{1, \alpha}\left(\bar{\Omega} ; \mathbb{R}^{3}\right)\right)$ be defined by

$$
\begin{gathered}
\tilde{w}^{i}\left(t_{i}\right)=\operatorname{curl} \aleph^{i}, \\
\partial_{t} \tilde{w}^{i}+(\tilde{u} \cdot \nabla) \tilde{w}^{i}=\left(\tilde{w}^{i} \cdot \nabla\right) \nabla \tilde{u}-\tilde{u}\left(\operatorname{div} \tilde{w}^{i}\right) \text { in } \tilde{\Omega} \times\left[t_{i}, t_{i+1}\right],
\end{gathered}
$$


where $\aleph^{i}$ is defined in Lemma 2.3 (see (2.21)). Here precisely, will be needed the fact that $\nu_{0}<\bar{\nu}$, in such a way that

$$
\operatorname{Supp} \tilde{w}^{i}\left(\cdot, t_{i+1}\right) \subset \tilde{\Omega} \backslash \bar{\Omega}
$$

Then, we define the applications $\zeta^{i}$ (which differ from those in Lemma 2.3 by the time intervals only), for $i=1, \ldots, s$, respectively on the intervals $\left[t_{i+k-1}, t_{i+k}\right]$ by the relations:

$$
\begin{gathered}
\operatorname{curl} \zeta^{i}=\tilde{w}^{i} \text { in } \bar{\Omega} \times\left[t_{i+k-1}, t_{i+k}\right], \\
\operatorname{div} \zeta^{i}=0 \text { in } \bar{\Omega} \times\left[t_{i+k-1}, t_{i+k}\right], \\
\zeta^{i} \cdot n=\partial_{n} \theta^{i} \text { on } \partial \Omega \times\left[t_{i+k-1}, t_{i+k}\right], \\
\int_{\Omega} \zeta^{i}\left(t_{k+i-1}\right) \cdot \mathcal{Q}_{j} d x=0, \\
\int_{\Omega}\left(\partial_{t} \zeta^{i}+u \wedge \operatorname{curl}\left(\zeta^{i}\right)\right) \cdot \mathcal{Q}_{j} d x=0, \quad \forall j \in\{1, \ldots, s\}, \forall t \in\left[t_{i+k-1}, t_{i+k}\right] .
\end{gathered}
$$

We can then define:

$$
\omega(x, t)=-\lambda_{i}\left(\frac{1}{2}\right) \tilde{w}^{i}(x, t) \quad \text { in }\left[t_{i}, t_{i+1}\right) \times \bar{\Omega}, \quad \forall i \in\{1, \ldots, s\} .
$$

As in $\left[\frac{1}{4}, \frac{1}{2}\right], \omega$ is continuous in variable $t$ from $\bigcup_{i=k}^{k+s-1}\left(t_{i}, t_{i+1}\right)$ to $C^{1, \alpha}(\bar{\Omega}) ; \mathbb{R}^{3}$, and with a limit in $\left(C^{1, \alpha}(\bar{\Omega}) ; \mathbb{R}^{3}\right)$ at the left and at the right of each $t_{i}$ (for $i \in\{k+1, \ldots, s+k-1\}$ ). Moreover, also as in $\left[\frac{1}{4}, \frac{1}{2}\right]$, it is continuous from $[1 / 2,3 / 4]$ into $C^{1, \alpha}(\bar{\Omega}) ; \mathbb{R}^{3}$.

Now we extend formula $(3.26-3.29)$ to the whole interval $[0,1]$ :

$$
\left\{\begin{array}{l}
\operatorname{curl} v=\omega \text { in } \Omega \times[0,1] \\
\operatorname{div} v=0 \text { in } \Omega \times[0,1] \\
v \cdot n=\mu(t) y_{0} \cdot n+\bar{y} \cdot n \text { in } \partial \Omega \times[0,1] \\
\int_{\Omega} v \cdot \mathcal{Q}_{i} d x=0, \forall t \in[0,1]
\end{array}\right.
$$

and also extend formula (3.32) (and hence extend the functions $\lambda_{i}$ ), in addition to (3.31):

$$
\int_{\Omega}\left(\partial_{t} v+\sum_{j=1}^{j=s} \lambda_{j}^{\prime}(t) \mathcal{Q}_{j}+\tilde{u} \wedge \omega\right) \cdot \mathcal{Q}_{i} d x=0, \quad \forall i \in\{1, \ldots, s\}, \forall t \in[0,1]
$$

We can now define $F(u)$ from $\bar{\Omega} \times[0,1]$ into $\mathbb{R}^{3}$ :

$$
F(u):=\left\{\begin{array}{l}
v+\sum_{i=1}^{s} \lambda_{i}(t) \mathcal{Q}_{i}(x) \text { in } \bar{\Omega} \times\left[0, \frac{3}{4}\right], \\
0 \text { in } \bar{\Omega} \times\left[\frac{3}{4}, 1\right] .
\end{array}\right.
$$

By this way, the function $F$ is correctly defined.

By (3.24) and (3.35) we get that

$$
\operatorname{curl} F(u) \in C^{0}\left([0,1] ; C^{1, \alpha}(\bar{\Omega}) ; \mathbb{R}^{3}\right) .
$$


By $(3.36-3.40,3.41)$ and $(3.43)$, we get that

$$
\lambda_{i}\left(\frac{3}{4}\right)=0
$$

Together with (3.45), this proves that

$$
F(u) \in C\left([0,1], C^{2, \alpha}(\bar{\Omega}) ; \mathbb{R}^{3}\right)
$$

and that the relation $(3.25)$ holds in $\Omega \times[0,1]$. Moreover, $F(u)$ is obviously a solution to the controllability problem (3.9).

\subsection{Proof of Lemma 3.1}

As it can be seen from (3.12), div $W$ satisfies the equation

$$
\partial_{t}(\operatorname{div} W)+(U \cdot \nabla)(\operatorname{div} W)=-(\operatorname{div} U)(\operatorname{div} W)
$$

The point (3.13) is hence clear.

To get the second point (3.14), we need more that (3.13). Let us indeed introduce the following family of special functions of $\Omega$. Let us consider, when $\partial \Omega$ has many connected components (that is in the case where $\left.H_{2}(\Omega) \neq 0\right)$, the set of functions $\mathcal{P}^{j}$ constructed as follows. We note the connected components of $\partial \Omega$ : $\gamma_{0}, \ldots$, $\gamma_{\bar{s}}$, and we define $\mathcal{P}^{j}:=\nabla \mathfrak{p}^{j}$ for any $j \in\{1, . ., \bar{s}\}$ where $\mathfrak{p}^{j}$ is defined by the relations

$$
\left\{\begin{array}{l}
\Delta \mathfrak{p}^{j}=0 \text { in } \Omega \\
\mathfrak{p}_{\mid \partial \Omega}=0 \text { on }\left(\partial \Omega \backslash \gamma_{j}\right), \\
\mathfrak{p}_{\mid \partial \Omega}=1 \text { on } \gamma_{j} .
\end{array}\right.
$$

It is well known that a solenoidal vector field on $\Omega$ can be written as the sum of the curl of a vector field and of a linear combination of the $\mathcal{P}^{j}$.

Consequently, if $\Omega$ is an open set such that $H_{2}(\Omega) \neq 0$, in order that (3.14) occurs, we need, besides the divergence free condition, the following relations to hold:

$$
\int_{\Omega} W(\cdot, t) \cdot \mathcal{P}^{j} d x=0, \quad \forall t \in[0, T], \quad \forall j \in\{1, . ., \bar{s}\}
$$

where we defined $\bar{s}$ and $\mathcal{P}^{j}$ in (3.48). These relations (3.49) are true for $t=0$, since $W(\cdot, 0)=$ curl $V_{0}$. We want to show this property stays true after $t=0$. To prove it, we compute (indices $j$ for $\mathcal{P}^{j}$ are dropped),

$$
\frac{d}{d t} \int_{\Omega} W . \nabla \mathfrak{p} d x=\int_{\Omega}\{(U . \nabla) W\} . \nabla \mathfrak{p} d x-\int_{\Omega}\{(W . \nabla) U\} . \nabla \mathfrak{p} d x=\int_{\Omega} U^{i} W_{i}^{j} \mathfrak{p}_{j} d x-\int_{\Omega} W^{i} \cdot U_{i}^{j} \mathfrak{p}_{j} d x
$$

where we denote derivations by lower indices and vector coordinates by upper exponents. Then, integrating by parts, we obtain, since $\operatorname{div} U=\operatorname{div} W=0$,

$$
\frac{d}{d t} \int_{\Omega} W \cdot \nabla \mathfrak{p} d x=\int_{\partial \Omega}\left(W^{i} U^{j} \mathfrak{p}_{j} n^{i}-W^{j} \mathfrak{p}_{j} U^{i} n^{i}\right) d \sigma
$$

As $\mathfrak{p}$ is constant on each connected component of $\partial \Omega, \nabla \mathfrak{p}$ is normal to the boundary everywhere on the boundary. We can deduce from this fact, that $(W \cdot n)(U . \nabla \mathfrak{p})=(W . \nabla \mathfrak{p})(U . n)$ on the boundary. The term on the right hand side of (3.50) is thus 0, so (3.49) stays true for all times. From that, we deduce that $W(\cdot, t)$ is a curl in $\Omega$. 


\section{Proof of Proposition 1.3}

\subsection{Introduction}

The goal of this section is to prove that $F$ admits a fixed point, and then to prove that it gives a proper solution to Proposition 1.3.

The first part of this proof is thus to find a set invariant by $F$.

We denote by $\mathcal{B}(B)$ the ball in $C^{0}\left([0,1], C^{2, \alpha}\left(\bar{\Omega} ; \mathbb{R}^{3}\right)\right)$ with radius $B$ and center 0 . Then this invariant set will be found as a certain $X_{\nu} \cap \mathcal{B}(B)$, for proper $B$ and $\nu$.

In a first step, we prove the following proposition:

Proposition 4.1. For any $B>0$, there exists $\nu_{0}$ and $\nu_{1}$, such that if one has $\left\|y_{0}\right\|_{1, \alpha, \Omega}<\nu_{1}$, then for all $\nu<\nu_{0}$, for all $u \in X_{\nu} \cap \mathcal{B}(B)$, one has $F(u) \in X_{\nu}$.

In a second step we prove this proposition:

Proposition 4.2. There exists $\nu_{2}>0$, such that if $\left\|y_{0}\right\|_{C^{2, \alpha}\left(\bar{\Omega} ; \mathbb{R}^{3}\right)} \leq \nu_{2}$, and if we define the sequences of functions $\left(y^{m}\right)_{m \geq 0} \in\left(C^{0}\left([0,1], C^{2, \alpha}\left(\bar{\Omega} ; \mathbb{R}^{3}\right)\right)\right)^{\mathbb{N}}$ and $\left(\omega^{m}\right)_{m \geq 0} \in\left(C^{0}\left([0,1], C^{1, \alpha}\left(\bar{\Omega} ; \mathbb{R}^{3}\right)\right)\right)^{\mathbb{N}}$ as follows:

$$
\left\{\begin{array}{l}
y^{0}(x, t)=\mu(t) y_{0}(x)+\bar{y}(x, t), \\
y^{m+1}=F\left(y^{m}\right), \\
\omega^{m+1} \text { defined as previously on } \tilde{\Omega} \times[0,1]
\end{array}\right.
$$

then the sequence $\left(y^{m}\right)_{m \geq 0}$ is bounded in $C^{0}\left([0,1], C^{2, \alpha}\left(\bar{\Omega} ; \mathbb{R}^{3}\right)\right)$, the bound depending only on $\Omega$ and $\nu_{2}$.

$A$ fortiori, we will be able to find $B$ and $\nu$ such that

$$
F\left(X_{\nu} \cap \mathcal{B}(B)\right) \subset X_{\nu} \cap \mathcal{B}(B) .
$$

The last step of the proof of Proposition 1.3 is then to establish that $F$ has a fixed point solution to the non-linear controllability problem.

The proofs of these propositions will require a technique introduced by Bardos and Frisch in [1]. Particularly, we will use the following lemma:

Lemma 4.3. ([1], Lem. 1) Let $u, v$ and $g$ be three functions of regularity $C^{0}\left([0, T], C^{1, \alpha}\left(\tilde{\Omega}, \mathbb{R}^{3}\right)\right)$, satisfying the relations

$$
\frac{\partial u}{\partial t}+(v \cdot \nabla) u=g, v \cdot n_{\mid \partial \tilde{\Omega} \times[0, T]}=0 .
$$

Then we have on $[0, T]$

$$
\frac{d}{d t^{+}}\|u\|_{0, \alpha, \tilde{\Omega}} \leq\left\|\partial_{t} u\right\|_{0, \alpha, \tilde{\Omega}} \leq\|g\|_{0, \alpha, \tilde{\Omega}}+\alpha\|\nabla v\|_{0, \alpha, \tilde{\Omega}} \cdot\|u\|_{0, \alpha, \tilde{\Omega}} .
$$

\subsection{Proof of Proposition 4.1}

In the sequel, we will denote by $C, C^{\prime}, C_{1}$ and $C_{2}$ different positive constants depending only on $\Omega$.

In this section, we will mark each object introduced in the previous section and corresponding to the $m$-th iteration of the operator $F$ in the construction of the sequence $\left(y^{m}\right)_{m \geq 0}$ by a lower index $m$.

As previously, we will first consider $t$ in the set $[0,1 / 2]$, and then $t$ in the interval $[1 / 2,1]$. 
When considering the $w^{m}$ introduced in Section 3, we will no longer use the upper index $l$ (corresponding to the $l$-th ball $\left.B\left(x_{l}, r_{l}\right)\right)$ in order not to confuse with the index $m$ corresponding to this $m$-th iteration. All the assertions about $w$ will be valid for any upper index. We will do the same with the index " $i$ " in $\lambda_{i}$. Also, when considering a time-dependent function $f:=f(x, t)$, we will make no difference between $\|f(\cdot, t)\|$ and $\|f\|(t)$, whatever spatial norm we use.

We first get an estimate on $\omega_{m+1}^{*}$. By (3.15) and Lemma 4.3, one easily gets for $t \in[0,1]$

$$
\frac{d}{d t^{+}}\left\|\omega_{m+1}^{*}\right\|_{0, \alpha, \Omega}(t) \leq(2+\alpha)\left\|\tilde{y}^{m}\right\|_{1, \alpha, \tilde{\Omega}}(t)\left\|\omega_{m+1}^{*}\right\|_{0, \alpha, \Omega}(t)
$$

With Gronwall's lemma, we deduce from (4.4) that for $t \in[0,1]$

$$
\left\|\omega_{m+1}^{*}\right\|_{0, \alpha, \Omega}(t) \leq\left\|\omega_{m+1}^{*}\right\|_{0, \alpha, \Omega}(0) e^{(2+\alpha) t\left\|y^{\tilde{m}}\right\|_{C^{0}\left([0,1], C^{1, \alpha}(\bar{\Omega}) ; \mathbb{R}^{3}\right)}}
$$

We do the same with the equation (3.17), and get by Lemma 4.3 the estimate for $t \in[1 / 4,1 / 2]$

$$
\frac{d}{d t^{+}}\left\|w_{m+1}\right\|_{0, \alpha, \Omega}(t) \leq(2+\alpha)\left\|\tilde{y}^{m}\right\|_{1, \alpha, \tilde{\Omega}}\left\|w_{m+1}\right\|_{0, \alpha, \Omega}(t)
$$

We easily deduce from that and from Gronwall's lemma that for $t \in[1 / 4,1 / 2]$

$$
\left\|w_{m+1}\right\|_{0, \alpha, \Omega}(t) \leq\left\|w_{m+1}\right\|_{0, \alpha, \Omega}\left(\frac{1}{4}\right) e^{(2+\alpha)\left(t-\frac{1}{4}\right)\left\|\tilde{y}^{m}\right\|_{C^{0}\left([0,1], C^{1, \alpha}(\bar{\Omega}) ; \mathbb{R}^{3}\right)}}
$$

from which we get, with $(3.24)$, that for $t \in[1 / 4,1 / 2]$

$$
\left\|\omega^{m+1}\right\|_{0, \alpha, \Omega}(t) \leq k\left\|\omega^{m}\right\|_{0, \alpha, \Omega}\left(\frac{1}{4}\right) e^{3\left(t-\frac{1}{4}\right)\left\|\tilde{y}^{m}\right\|_{C^{0}\left([0,1], C^{1, \alpha}(\bar{\Omega}) ; \mathbb{R}^{3}\right)}},
$$

from what we deduce with (3.15) and (4.5) that for $t \in[0,1 / 2]$

$$
\left\|\omega^{m+1}\right\|_{0, \alpha, \Omega}(t) \leq k\left\|y_{0}\right\|_{1, \alpha, \Omega} e^{3 t\left\|\tilde{y}^{m}\right\|_{C^{0}\left([0,1], C^{1, \alpha}(\bar{\Omega}) ; \mathbb{R}^{3}\right)}}
$$

We now want to get (4.9) for the rest of the time $[1 / 2,1]$, and by the way obtain an estimate on the $\lambda_{i}^{m}$. By (3.42) and (3.43) we have on $[0,1]$

$$
\frac{d}{d t^{+}}\left|\lambda_{i}^{m}\right| \leq C\left\|\omega^{m+1}\right\|_{0, \Omega}\left\|y^{m}\right\|_{0, \Omega}
$$

We deduce that for $t \in[0,1 / 2]$

$$
\left|\lambda_{i}^{m+1}(t)\right| \leq C_{1}\left\|y_{0}\right\|_{1, \alpha, \Omega}\left\|y^{m}\right\|_{C^{0}\left([0,1], C^{1}(\bar{\Omega}) ; \mathbb{R}^{3}\right)} e^{3\left\|\tilde{y}^{m}\right\|_{C^{0}\left([0,1], C^{1}(\bar{\Omega}) ; \mathbb{R}^{3}\right)}}
$$

As for (4.5), one can deduce that for all $i \in\{k, \ldots, k+s-1\}$ and for all $t \in\left[t_{i}, t_{i+1}\right)$, one has

$$
\left\|\omega^{m+1}\right\|_{0, \alpha, \Omega}(t) \leq\left\|\omega^{m+1}\right\|_{0, \alpha, \Omega}\left(t_{i}\right) e^{(2+\alpha)\left(t-t_{i}\right)\left\|y^{\tilde{m}}\right\|_{C^{0}\left([0,1], C^{1, \alpha}(\bar{\Omega}) ; \mathbb{R}^{3}\right)}} .
$$

With (3.41) and (4.11), we get that for $t \in[1 / 2,3 / 4]$

$$
\left\|\omega^{m+1}\right\|_{0, \alpha}(t) \leq C_{2}\left\|y_{0}\right\|_{1, \alpha, \Omega}\left\|y^{m}\right\|_{C^{0}\left([0,1], C^{1}(\bar{\Omega})\right)} e^{3\left\|\tilde{y}^{m}\right\|_{C^{0}\left([0,1], C^{1}(\bar{\Omega}) ; \mathbb{R}^{3}\right)}} .
$$


We can deduce from it, with $(3.42-3.44,4.9,4.11)$ and that for $t \in[0,1]$

$$
\|F(u)-\bar{y}\|_{0, \Omega}(t) \leq C\left(\left\|y^{m}\right\|_{1, \alpha, \Omega}\right)\left\|y_{0}\right\|_{1, \alpha, \Omega},
$$

where $C(\cdot)$ is an increasing, positive real-valued, numerical function.

So we have proved that $F$ is well defined on $X_{\nu}$ and that for any $B>0$, there exists $\nu_{1}=\nu_{1}(B)>0$ such that for any $y_{0}$ satisfying $\left\|y_{0}\right\|_{2, \alpha, \Omega}<\nu<\nu_{1}$, one has

$$
F\left(X_{\nu} \cap \mathcal{B}^{C\left([0,1], C^{1, \alpha}\left(\bar{\Omega} ; \mathbb{R}^{3}\right)\right)}(B)\right) \subset X_{\nu}
$$

where we have denoted by $\mathcal{B}^{C\left([0,1], C^{1, \alpha}\left(\bar{\Omega} ; \mathbb{R}^{3}\right)\right)}(B)$ the 0 -centered open ball in $C\left([0,1], C^{1, \alpha}\left(\bar{\Omega} ; \mathbb{R}^{3}\right)\right)$ with radius $B$, at least if $\nu_{0}$ and $\nu_{1}$ (depending on $B$ ) are small small enough.

\subsection{Proof of Proposition 4.2}

Let us consider the sequence $\left(y^{m}\right)_{m \geq 0} \in\left(C^{0}\left([0,1], C^{2, \alpha}\left(\bar{\Omega} ; \mathbb{R}^{3}\right)\right)\right)^{\mathbb{N}}$ by $(4.1)$.

In a first step, we just deal with the boundedness of the sequence $\left(y^{m}\right)$ in the space $C^{0}\left([0,1], C^{1, \alpha}\left(\bar{\Omega} ; \mathbb{R}^{3}\right)\right)$. We will come back to the boundedness in $C^{0}\left([0,1], C^{2, \alpha}\left(\bar{\Omega} ; \mathbb{R}^{3}\right)\right)$ at the end of this section.

Let us denote by $C_{i}, i \geq 1$, various constants which do not depend on $m$. Combining (4.9) and (4.12), one can get for any $t \in[0,1]$ that

$$
\left\|\omega^{m+1}\right\|_{0, \alpha, \Omega} \leq C_{3}\left\|y_{0}\right\|_{1, \alpha, \Omega} e^{3\left\|y^{m}\right\|_{C^{0}\left([0,1], C^{1, \alpha}(\bar{\Omega}) ; \mathbb{R}^{3}\right)}}\left(1+\left\|y^{m}\right\|_{C^{0}\left([0,1], C^{1, \alpha}(\bar{\Omega}) ; \mathbb{R}^{3}\right)}\right) .
$$

By (4.10) and (4.12), one as also in $[0,1]$

$$
\left|\lambda_{i}^{m+1}\right| \leq C_{4}\left\|y_{0}\right\|_{1, \alpha, \Omega} e^{3\left\|y^{m}\right\|_{C^{0}\left([0,1], C^{1, \alpha}(\bar{\Omega}) ; \mathbb{R}^{3}\right)}}\left(1+\left\|y^{m}\right\|_{C^{0}\left([0,1], C^{1, \alpha}(\bar{\Omega}) ; \mathbb{R}^{3}\right)}^{2}\right) .
$$

On another side, by (3.42) and (3.44), one can find some constants such that, for any $t \in[0,1]$,

$$
\left\|y^{m+1}\right\|_{1, \alpha, \Omega}(t) \leq C_{7}\left\|y_{0}\right\|_{1, \alpha, \Omega}+C_{8}\left\|\omega^{m+1}\right\|_{0, \alpha}(t)+C_{9} \sum_{i}\left\|\lambda_{i}\right\|_{C^{0}([0,1])} .
$$

We deduce from $(4.14,4.15)$ and $(4.16)$ that for every $t \in[0,1]$

$$
\left\|y^{m+1}\right\|_{1, \alpha, \Omega}(t) \leq C_{10}\left\|y_{0}\right\|_{1, \alpha, \Omega}\left(1+e^{3\left\|y^{m}\right\|_{C^{0}\left([0,1], C^{1, \alpha}(\bar{\Omega}) ; \mathbb{R}^{3}\right)}}\right)\left(1+\left\|y^{m}\right\|_{C^{0}\left([0,1], C^{1, \alpha}(\bar{\Omega}) ; \mathbb{R}^{3}\right)}^{2}\right) .
$$

Note that this is made valid in $[3 / 4,1]$ because of the trivial form of $y^{m}$ in this time segment.

We want to deduce from (4.17) that, reducing $\nu_{0}$ if necessary, one can get

$$
\left\|y^{m}\right\|_{C^{0}\left([0,1], C^{1, \alpha}(\Omega) ; \mathbb{R}^{3}\right)} \leq 2\|\bar{y}\|_{C^{0}\left([0,1], C^{1, \alpha}(\Omega) ; \mathbb{R}^{3}\right)}, \quad \forall m \in \mathbb{N} .
$$

The proof of (4.18) is done by induction.

We check (4.18) for $m=0$. As $y^{0}=\mu(t) y_{0}+\bar{y}$, (4.18) is satisfied if $\nu_{0}<\|\bar{y}\|_{1, \alpha}$.

We now suppose that (4.18) is satisfied for a fixed $m$, and show it is still valid at rank $m+1$.

We impose $\nu_{0}$ in order that

$$
C_{10} \nu_{0}\left(1+e^{6\|\bar{y}\|_{C^{0}\left([0,1], C^{1, \alpha}(\bar{\Omega}) ; \mathbb{R}^{3}\right)}}\right)\left(1+4\|\bar{y}\|_{C^{0}\left([0,1], C^{1, \alpha}(\bar{\Omega}) ; \mathbb{R}^{3}\right)}^{2}\right)<2\|\bar{y}\|_{C^{0}\left([0,1], C^{1, \alpha}(\bar{\Omega}) ; \mathbb{R}^{3}\right)} .
$$


Using (4.17) and the induction hypothesis, we get (4.18) at rank $m+1$. Consequently, we have a bound on the sequence $\left(\left\|y^{m}\right\|_{1, \alpha}\right)_{m \geq 0}$. Hence, we can choose a "universal" $\nu_{1}$ so that actually $y^{m}$ belongs to $X_{\nu}$ at each step.

Now we prove the boundedness of the sequence $\left(y^{m}\right)$ in $C^{0}\left([0,1], C^{2, \alpha}(\bar{\Omega}) ; \mathbb{R}^{3}\right)$.

The proof is quite the same as for the $C^{1, \alpha}$ bound. It consists in the majoration of $\left\|y^{m+1}\right\|_{C^{2, \alpha}}$ by a factor of the form $\left\|y_{0}\right\|_{2, \alpha} C\left(\left\|y^{m}\right\|_{2, \alpha}\right)$.

We already have this type of bound on the $\lambda_{i}$. It is hence sufficient to have such a bound for $\left\|\omega^{m}\right\|_{C^{2, \alpha}}$.

As previously, it is consequently sufficient to get a $C^{0}\left([0,1], C^{2, \alpha}(\tilde{\Omega}) ; \mathbb{R}^{3}\right)$ bound for the $\left(w^{m}\right)$. But considering the derivatives of the relation (3.17) and using Lemma 4.3, we get terms in the right hand side all majored either by a certain $\left\|\tilde{y}^{m}\right\|_{2, \alpha}\left\|w^{m+1}\right\|_{1, \alpha}$, or by a constant because we know that $\left(y^{m}\right)$ is bounded in $C^{0}\left([0,1], C^{1, \alpha}(\tilde{\Omega}) ; \mathbb{R}^{3}\right)$.

Hence, the same demonstration works again, if $\left\|y_{0}\right\|_{2, \alpha}$ is chosen small enough.

\subsection{Proof of Proposition $\mathbf{1 . 3}$}

Now we prove the convergence of the sequences $\left(y^{m}\right)$ and $\left(\omega^{m}\right)$. We first show the convergence on the interval $[0,1 / 2]$. We still follow [1], and aim at proving that the sequence $\left(\omega^{m}, \lambda^{m}\right)$ satisfies the Cauchy criterion in $\cap_{i=0}^{i=k} C^{0}\left(\left[t_{i-\frac{1}{2}}, t_{i+\frac{1}{2}}\right], C^{0, \alpha}(\tilde{\Omega}) ; \mathbb{R}^{3} \times \mathbb{R}^{s}\right)$.

For all times except $t_{i+\frac{1}{2}}, i \in\{0, \ldots, k-1\}$, we have

$$
\begin{aligned}
\partial_{t}\left(\omega^{p}-\omega^{m}\right) & +\left(\tilde{y}^{m-1} \cdot \nabla\right)\left(\omega^{p}-\omega^{m}\right)=\left[\left(\tilde{y}^{p-1}-\tilde{y}^{m-1}\right) \cdot \nabla\right] \omega^{p}+\left[\left(\omega^{p}-\omega^{m}\right) \cdot \nabla\right] \tilde{y}^{p-1} \\
& +\left(\omega^{m} \cdot \nabla\right)\left(\tilde{y}^{p-1}-\tilde{y}^{m-1}\right)-\left(\operatorname{div} \tilde{y}^{p-1}\right)\left(\omega^{p}-\omega^{m}\right)-\left(\operatorname{div} \tilde{y}^{m-1}-\operatorname{div} \tilde{y}^{p-1}\right) \omega^{m} .
\end{aligned}
$$

Any term at the right of this equality can be bounded in norm $\|\cdot\|_{0, \alpha, \tilde{\Omega}}$ (within a multiplicative constant) either by the norm $\left\|\omega^{p}-\omega^{m}\right\|_{0, \alpha, \tilde{\Omega}}(t)$, or by the norm $\left\|\tilde{y}^{p-1}-\tilde{y}^{m-1}\right\|_{1, \alpha, \tilde{\Omega}}(t)$.

With the help of lemma 4.3 , we deduce that for $t \in\left[\frac{1}{4}, \frac{1}{2}\right]$, except at times $t_{i+\frac{1}{2}}$, one has

$$
\left\|\partial_{t}\left(\omega^{p}-\omega^{m}\right)\right\|_{0, \alpha, \tilde{\Omega}}(t) \leq C\left(\left\|\tilde{y}^{p-1}-\tilde{y}^{m-1}\right\|_{0, \alpha, \tilde{\Omega}}(t)+\left\|\omega^{p}-\omega^{m}\right\|_{0, \alpha, \tilde{\Omega}}(t)\right),
$$

and consequently

$$
\left\|\partial_{t}\left(\omega^{p}-\omega^{m}\right)\right\|_{0, \alpha, \tilde{\Omega}}(t) \leq C\left(\left\|y^{p-1}-y^{m-1}\right\|_{1, \alpha, \Omega}(t)+\left\|\omega^{p}-\omega^{m}\right\|_{0, \alpha, \tilde{\Omega}}(t)\right),
$$

which gives finally

$$
\left\|\partial_{t}\left(\omega^{p}-\omega^{m}\right)\right\|_{0, \alpha, \tilde{\Omega}}(t) \leq C\left(\left\|\omega^{p}-\omega^{m}\right\|_{0, \alpha, \tilde{\Omega}}(t)+\left\|\omega^{p-1}-\omega^{m-1}\right\|_{0, \alpha, \tilde{\Omega}}(t)+\left|\lambda^{m-1}-\lambda^{p-1}\right|(t)\right) .
$$

As the expression (4.20) is also valid when replacing $w$ by $\omega^{*}$, one can get that (4.21) is valid in fact in $\left[0, t_{\frac{1}{2}}\right]$.

We do the same for $\lambda_{i}^{m}-\lambda_{i}^{p}$ and get the same way, using (3.43), that

$$
\left|\partial_{t}\left(\lambda^{p}-\lambda^{m}\right)\right|(t) \leq C\left(\left\|\omega^{p}-\omega^{m}\right\|_{1, \alpha, \tilde{\Omega}}(t)+\left\|y^{p-1}-y^{m-1}\right\|_{1, \alpha, \Omega}\right)(t),
$$

and hence that

$$
\left|\partial_{t}\left(\lambda^{p}-\lambda^{m}\right)\right|(t) \leq C\left(\left\|\omega^{p}-\omega^{m}\right\|_{1, \alpha, \tilde{\Omega}}(t)+\left\|\omega^{p-1}-\omega^{m-1}\right\|_{1, \alpha, \Omega}(t)+\left|\lambda^{p-1}-\lambda^{m-1}\right|(t)\right) .
$$

Let us denote by $\mathcal{K}^{m, p}(t):=\left\|\omega^{p}-\omega^{m}\right\|_{1, \alpha, \tilde{\Omega}}(t)+\left|\lambda^{p}-\lambda^{m}\right|(t)$. 
Putting together (4.21) and (4.22), one gets

$$
\frac{d}{d t^{+}} \mathcal{K}^{m, p}(t) \leq C\left(\mathcal{K}^{m, p}(t)+\mathcal{K}^{m-1, p-1}(t)\right)
$$

By Gronwall's lemma, one gets, for $t \in\left[0, t_{\frac{1}{2}}\right]$

$$
\mathcal{K}^{m+1, p+1}(t) \leq C \int_{0}^{t} \mathcal{K}^{m, p}\left(t_{1}\right) e^{C t_{1}} d t_{1}
$$

and then by induction

$$
\mathcal{K}^{m+k, p+k}(t) \leq C^{k} \int_{0}^{t} \int_{0}^{t_{1}} \cdots \int_{0}^{t_{k-1}} \mathcal{K}^{m, p}\left(t_{k}\right) e^{C t_{1}+\cdots+C t_{k}} d t_{1} \ldots d t_{k} .
$$

Finally, one gets on $\left[0, t_{\frac{1}{2}}\right]$ :

$$
\mathcal{K}^{m+k, p+k}(t) \leq K \frac{e^{k C t}}{k !} \max _{s \in\left[0, t_{\frac{1}{2}}\right]} \mathcal{K}^{m, p}(s)
$$

from what we get the convergences of $\omega^{m}$ and $\lambda^{m}$ to some $\mathbf{w}$ and some $\bar{\lambda}$ for times in $\left[0, t_{\frac{1}{2}}\right]$ (to be more precise, the convergence are to be understood respectively in $C^{0}\left(\left[0, t_{\frac{1}{2}}\right], C^{0, \alpha}\left(\bar{\Omega} ; \mathbb{R}^{3}\right)\right)$ and in $\left.C^{0}\left(\left[0, t_{\frac{1}{2}}\right], \mathbb{R}^{s}\right)\right)$. Furthermore, these convergences determine those of $y^{m}$ and $w_{i}^{m}$ respectively in $C^{0}\left(\left[0, t_{\frac{1}{2}}\right], C^{1, \alpha}\left(\bar{\Omega} ; \mathbb{R}^{3}\right)\right)$ and $C^{0}\left(\left[0, t_{\frac{1}{2}}\right], C^{0, \alpha}\left(\bar{\Omega} ; \mathbb{R}^{3}\right)\right)$.

Consequently, we get the convergence of $\omega^{m}\left(t_{\frac{1}{2}}^{+}\right)$also, and we can repeat the same method during the interval $\left[t_{\frac{1}{2}}, t_{\frac{3}{2}}\right]:$ instead of $(4.24)$, we get

$$
\mathcal{K}^{m+1, p+1}(t) \leq C \int_{t_{\frac{1}{2}}}^{t} \mathcal{K}^{m, p}\left(t_{1}\right) e^{C t_{1}} d t_{1}+\mathcal{K}^{m+1, p+1}\left(t_{\frac{1}{2}}^{+}\right)-\mathcal{K}^{m, p}\left(t_{\frac{1}{2}}^{+}\right),
$$

which leads, for $t \in\left[t_{\frac{1}{2}}^{+}, t_{\frac{3}{2}}\right]$, to

$$
\mathcal{K}^{m+k, p+k}(t) \leq K \frac{e^{k C t}}{k !} \max _{s \in\left[t_{\frac{1}{2}}^{+}, t_{\frac{3}{2}}\right]} \mathcal{K}^{m, p}(s)+\mathcal{K}^{m+k, p+k}\left(t_{\frac{1}{2}}^{+}\right)-\mathcal{K}^{m, p}\left(t_{\frac{1}{2}}^{+}\right) .
$$

We get the convergence on the interval $\left[t_{\frac{1}{2}}+, t_{\frac{3}{2}}\right]$, and then step by step we get the convergences in $C^{0}\left(\left[t_{\frac{3}{2}}, t_{\frac{5}{2}}\right], C^{0, \alpha}\left(\overline{\tilde{\Omega}} ; \mathbb{R}^{3}\right)\right)$ and in $C^{0}\left(\left[t_{\frac{3}{2}}, t_{\frac{5}{2}}\right], \mathbb{R}^{s}\right)$, etc.

Remains the problem of convergence in $[1 / 2,3 / 4]$.

Clearly, the convergence of $\left(\omega^{m}\right)$ and the one of $\left(y^{m}\right)$ on the interval $[0,1 / 2]$ determine the one of the $\lambda_{i}^{m}(1 / 2)$ to a certain $s$-uplet $\bar{\lambda}(1 / 2) \in \mathbb{R}^{s}$.

As $\omega$ and $\lambda$ are governed by the same equations as during $\left[0, \frac{1}{2}\right]$, the result $(4.23)$ is also valid for $t \in\left[\frac{1}{2}, 1\right]$, except at times $t_{i}$, with $i \in\{k+1, k+s+1\}$. One consequently deduces the same convergence result in intervals $\left[t_{i}, t_{i+1}\right]$ for $i$ in $\{k+1, k+s+1\}$. Finally, $y^{m}$ and $\omega^{m}$ do converge to some $y$ and $\mathbf{w}$ in $C^{0}\left([0,1], C^{1, \alpha}\left(\bar{\Omega} ; \mathbb{R}^{3}\right)\right)$ and $C^{0}\left([0,1], C^{0, \alpha}\left(\bar{\Omega} ; \mathbb{R}^{3}\right)\right)$.

Let us now prove the fixed point $y$ is a solution to our controllability problem. 
From $(3.25,3.42)$ and $(3.44)$, we get that

$$
\left\{\begin{array}{l}
\operatorname{div} y=0 \text { in } \Omega \times[0,1] \\
y \cdot n=0 \text { on }\left(\partial \Omega \backslash \Gamma_{0}\right) \times[0,1] \\
\operatorname{curl} y=\mathbf{w} \text { in } \Omega \times[0,1] \\
\partial_{t} \mathbf{w}+(y \cdot \nabla) \mathbf{w}=(\mathbf{w} . \nabla) y \text { in } \Omega \times[0,1]
\end{array}\right.
$$

With (3.44, 3.32) and (4.26), we get (1.3) for some $p \in \mathfrak{D}^{\prime}(\Omega \times(0,1))$. From (3.22) (with (3.23)), we get

$$
\mathbf{w}(\cdot, 1)=0 .
$$

Together with (3.41), this leads to

$$
y(\cdot, 1)=0 .
$$

As (1.6) is obviously satisfied by $y$, we get a solution for Proposition 1.3.

\section{End of The PROOF OF TheOREM 1.1}

Here we deduce Theorem 1.1 from Proposition 1.3. Let us consider $y_{0}$ and $y_{1}$ two divergence-free elements of $C^{2, \alpha}\left(\bar{\Omega} ; \mathbb{R}^{3}\right)$. We use Proposition 1.3 and we obtain a certain $\nu$ by this proposition. For $y_{0}$, we choose $\epsilon$ in ]$\left(0, T / 2[)\right.$ small enough so that $\left|\epsilon y_{0}\right|_{2, \alpha}<\nu$. Proposition 1.3 for $\epsilon y_{0}$ give us a couple $(y, p)$. Then $(\tilde{y}, \tilde{p})$ defined by

$$
\begin{aligned}
& \tilde{y}(x, t)=\epsilon^{-1} y\left(x, \epsilon^{-1} t\right) \forall t \in[0, \epsilon], \\
& \tilde{p}(x, t)=\epsilon^{-2} p\left(x, \epsilon^{-1} t\right) \forall t \in[0, \epsilon], \\
& \tilde{y}(x, t)=0 \forall t \in[\epsilon, T / 2], \\
& \tilde{p}(x, t)=0 \forall t \in[\epsilon, T / 2],
\end{aligned}
$$

is still solution of the Euler system with $\tilde{y}_{\mid t=0}=y_{0}$. We operate similarly for $-y_{1}$, so that we obtain $\left(\tilde{y}^{\prime}, \tilde{p}^{\prime}\right)$. Then $\left(-\tilde{y}^{\prime}(T-t), \tilde{p}^{\prime}(T-t)\right)$ is again solution of Euler equation. The function chosen equal to $\tilde{y}^{\prime}$ on $[0, T / 2]$ and to $\tilde{y}^{\prime}(T-t)$ on $[T / 2, T]$, associated to the pressure function equal to $\tilde{p}$ on $[0, T / 2]$ and to $\tilde{p}^{\prime}(T-t)$ on $[T / 2, T]$, gives an answer to the problem.

\section{Proof of Lemma 2.1}

The proof is quite the same as the one of ([5], Lem. A.1), which proves the result for interior points.

Let us recall the lemma in [5]:

Lemma 6.1. ([5], Lem. A.2) For any $\bar{x}$ in $\Omega$,

$$
\left\{\nabla \theta(\bar{x}) ; \theta \in C^{\infty}(\bar{\Omega} ; \mathbb{R}), \Delta \theta=0 \text { in } \bar{\Omega} \text { and } \frac{\partial \theta}{\partial n}=0 \text { on } \partial \Omega \backslash \Gamma_{0}\right\}=\mathbb{R}^{3} .
$$

(The proof of Coron in [5] still holds for dimension 3 with $\Omega^{\#}$ of [5] defined as in Fig. 1.)

In this paper, we prove this result holds for a point $\bar{x}$ of the boundary as well: 


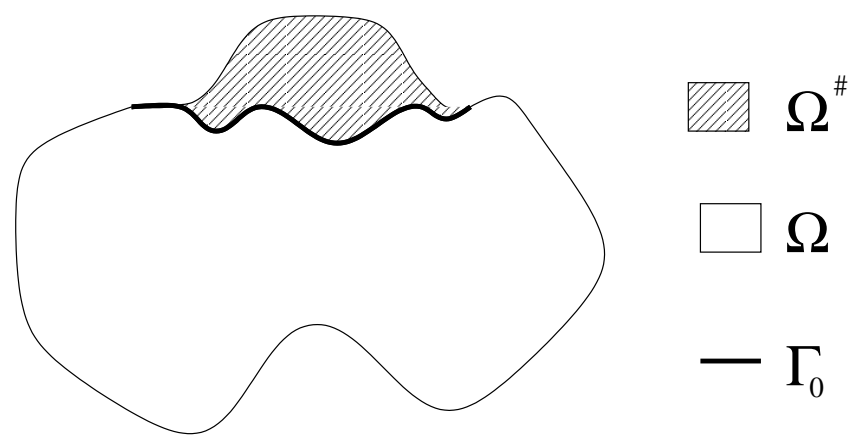

Figure 1. The enhanced domain.

Lemma 6.2. For any $\bar{x}$ in $\partial \Omega$, the set

$$
\left\{\nabla \theta(\bar{x}) ; \theta \in C^{\infty}(\bar{\Omega} ; \mathbb{R}) \text { s. t. } \Delta \theta=0 \text { in } \bar{\Omega} \text { and } \frac{\partial \theta}{\partial n}=0 \text { on } \partial \Omega \backslash \Gamma_{0}\right\}
$$

is equal to the tangent plane to $\partial \Omega$ at the point $\bar{x}$, which we will denote by $T_{\bar{x}}(\partial \Omega)$.

We will prove this lemma in section 8 .

We then follow [5]. We fix $\bar{x}$ in $\partial \Omega$ (the case when $\bar{x} \in \Omega$ has already been treated in [5]) and $\bar{y} \in \tilde{\Omega} \backslash \bar{\Omega}$. We choose some $F$ in $C^{\infty}([0,1], \tilde{\Omega})$, such that

$$
\begin{gathered}
F(t)=\bar{x}, \forall t \in\left[0, \frac{1}{4}\right], \\
F(t)=\bar{y}, \forall t \in\left[\frac{3}{4}, 1\right] \\
\frac{d F(t)}{d t} \in T_{F(t)} \partial \Omega \text { for } F(t) \in \partial \Omega \backslash \Gamma_{0} .
\end{gathered}
$$

It follows from the previous lemma that one can find $h_{1}, \ldots, h_{l}$ and $\xi^{1}, \ldots, \xi^{l}, 2 l$ functions respectively in $C^{\infty}([0,1], \mathbb{R})$ and in $C_{0}^{\infty}(\tilde{\Omega})$ such that

$$
\begin{aligned}
\operatorname{Supp} h_{i} & \subset\left[\frac{1}{4}, \frac{3}{4}\right], \forall i \in\{1, \ldots, l\}, \\
\Delta \xi^{i} & =0 \text { in } \Omega, \forall i \in\{1, \ldots, l\}, \\
\partial_{n} \xi^{i} & =0, \text { on } \partial \Omega \backslash \Gamma_{0}, \forall i \in\{1, \ldots, l\}, \\
\text { and } \phi^{\nabla \xi}(x, 0, t) & =F(t), \forall t \in[0,1],
\end{aligned}
$$

where

$$
\xi\left(x^{\prime}, t\right)=\sum_{i=1}^{l} h_{i}(t) \xi^{i}\left(x^{\prime}\right), \forall\left(x^{\prime}, t\right) \in \overline{\tilde{\Omega}} \times[0,1]
$$

So we found the desired function. 


\section{Proof of Lemma 2.3}

In order to prove Lemma 2.3, we will use vortex located on loops which we move around in the domain.

Note that, reducing $\Gamma_{0}$ if necessary, we can suppose it is a ball drawed in $\partial \Omega$ and which does not contain a loop which is non trivial in $\partial \Omega$.

We fix a certain $\mathbf{i}$ in $\{1, \ldots, s\}$. For that $\mathbf{i}$, we will construct a certain $\bar{y}^{\mathbf{i}}$, and a certain $\omega_{0}^{\mathbf{i}}$ such that finally the matrix

$$
\mathfrak{M}=\left(\int_{\Omega} \zeta^{i}(1) \cdot \mathcal{Q}_{j} d x\right)_{i=1 \ldots s, j=1 \ldots s}
$$

satisfies (2.29) where the functions $\zeta^{i}$ are defined by $(2.23-2.27)$.

We introduce a hypersurface $\Sigma_{\mathbf{i}}^{\prime}$ in $\Omega$, equivalent to $\Sigma_{\mathbf{i}}$ (in the sense that there exists a part $\Sigma_{\mathbf{i}}^{\prime \prime}$ of $\partial \Omega$ such that $\Sigma_{\mathbf{i}} \cup \Sigma_{\mathbf{i}}^{\prime} \cup \Sigma_{\mathbf{i}}^{\prime \prime}$ is topologically a sphere) and which boundary cuts $\Gamma_{0}$. In fact, we could have required directly from $\Sigma_{\mathbf{i}}$ to cut $\Gamma_{0}$.

In a first step, let us define a certain smooth vector field on $\mathbb{R}^{3}$, with compact support in time, which we will denote by $y^{\mathbf{i}}(x, t)$. Then we will define actually $\bar{y}^{\mathbf{i}}$ of the required form, so that it will be close enough to $y^{\mathbf{i}}$.

We consider a Jordan curve $J_{0}$ in $\Omega^{\#} \backslash \bar{\Omega}$, where $\Omega^{\#}$ is defined as previously (in Sect. 6 ). The vector field $y^{\mathbf{i}}$ will be chosen according to the trajectory of $J_{0}$ (or more precisely, of a part of $J_{0}$ ) inside $\bar{\Omega}$.

Along the construction of $y^{\mathrm{i}}$, we will denote by $J(t)$ the Jordan curve obtained as the image of $J_{0}$ by the flow of $y^{\mathbf{i}}$ between times 0 and $t$.

To clarify the required motion of $J_{0}$ by the flow of $y^{\mathbf{i}}$, we will represent it in the cross-section $\Sigma_{\mathbf{i}}^{\prime}$ of $\Omega$ described in Figure 2 (in the case of a simple torus).

We divide the time interval $\left[\frac{1}{4}, \frac{3}{4}\right]$ in three stages.

In a first step $y^{\mathbf{i}}$ makes $J_{0}$ partially enter inside $\Omega$ by the "hole" $\Gamma_{0}$ as described in the Figure 3 and such that $J \cap \Omega$ stays close to $\Sigma_{\mathbf{i}}^{\prime}$.

At the end of this first stage, $J(t)$ cuts $\Gamma_{0}$ at two points $a$ and $b$, and we will denote $\tilde{J}$ the part of $J(t)$ inside $\Omega$ at this moment.

In the second step, we demand that $a$ stays fixed, and make $b$ describe $\partial \Sigma_{\mathbf{i}}^{\prime}$ and also that $\tilde{J}$ "nearly" describes $\Sigma_{\mathbf{i}}^{\prime}$. We make this process continue until $b$ belongs again to $\Gamma_{0}$ such as described in Figure 4.

The last step of the movement consists in making $\tilde{J}$ leave $\bar{\Omega}$, staying close to $\Sigma_{\mathbf{i}}^{\prime}$, and taking care that $J(1)$ does no longer cut $\partial \Omega \backslash \Gamma_{0}$ (so that at the end of the processes, $J(1)$ "describes a loop around" $\Omega$ ). This step is described in Figure 5.

In conclusion, we could say that we have chosen $y^{\mathbf{i}}$ so that inside $\Omega, J(t)$ describes a surface $\tilde{\Sigma}_{\mathbf{i}}$ equivalent to $\Sigma_{\mathbf{i}}$

In fact, the process described in Figures 3-5 may be more complicated than it appears in the previous presentation. Indeed, there can be some obstacles "on the way" back of the vortex filament, during the step described by Figure 5. For example, one can think of a plain torus, inside which one has cut out another plain

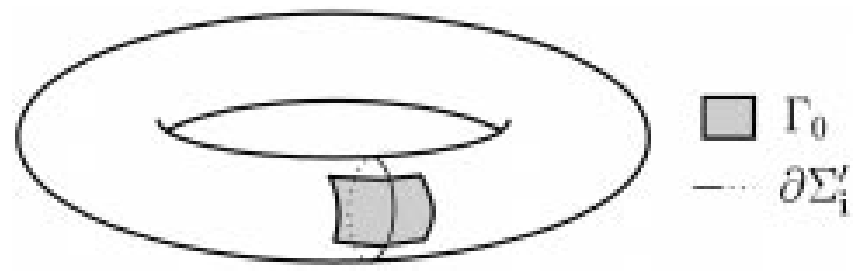

FIgURE 2. The cross-section from which the next figures are viewed. 

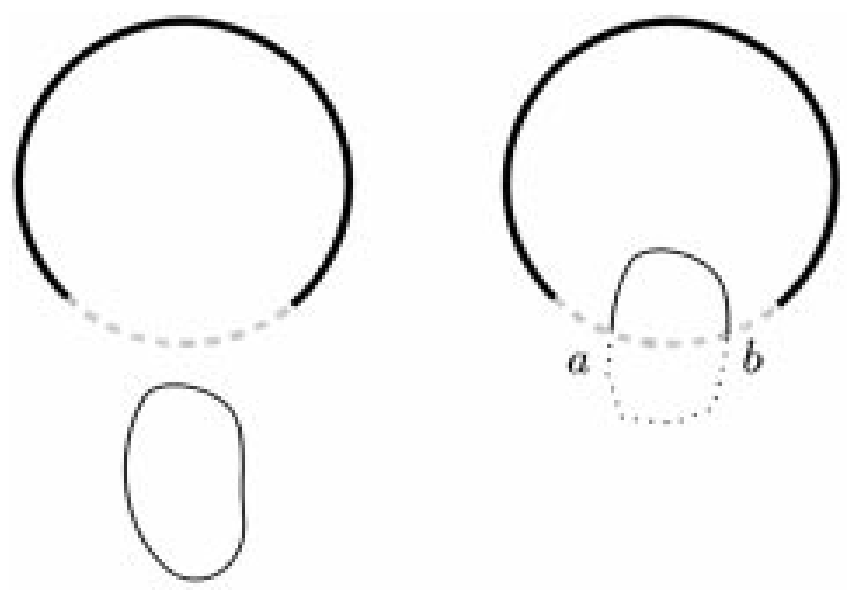

FiguRE 3. Entering $\Omega$.
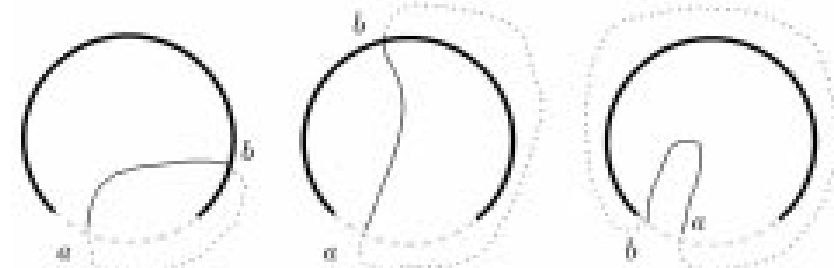

Figure 4. Describing $\Sigma_{\mathbf{i}}^{\prime}$ (or nearly it).
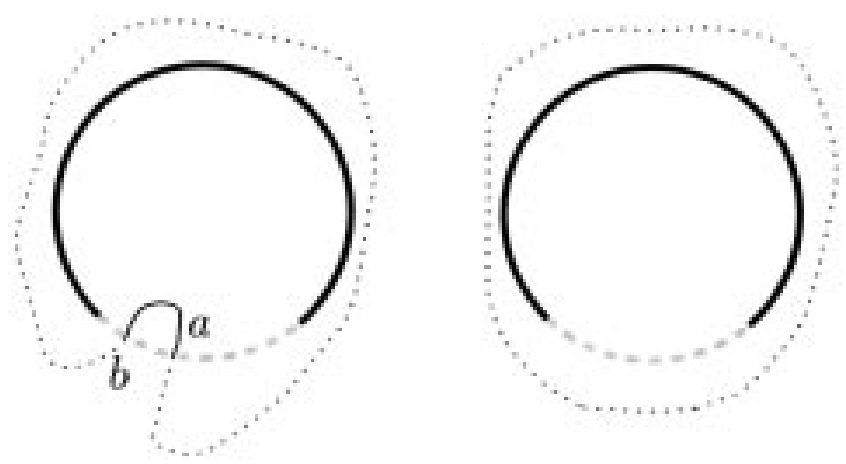

FiguRE 5. Leaving $\Omega$.

torus which winds around the "hole" of the first one (that is precisely, such that there is a generator - or a non-trivial element - of the fundamental group of the first torus represented in the second one). Then the filament must meet the internal torus during the process described by Figures 3-5. One can also think of the same domain, where one has "glued" the two tori by means of a cylinder. This domain raises the same problem, and moreover has a connected boundary. Let us denote it by $\mathcal{T}$. 
It appears that this kind of obstacle can be passed during the process, the same way as the filament passes the torus in Figures 3-5. This is made possible because the control zone encounters any connected component of the boundary.

We give in the following Figures 6-8 the example of a torus $\mathfrak{T}$, in which one has cut out an other plain torus $T_{1}$ and a domain of type $\mathcal{T}$, which both wind around the hole of $\mathfrak{T}$ (that is there exists in $T_{1}$ and in $\mathcal{T}$ some loops, non-trivial in $\mathfrak{T})$. The obtained domain is represented in color according to the section $\Sigma_{i}^{\prime}$.
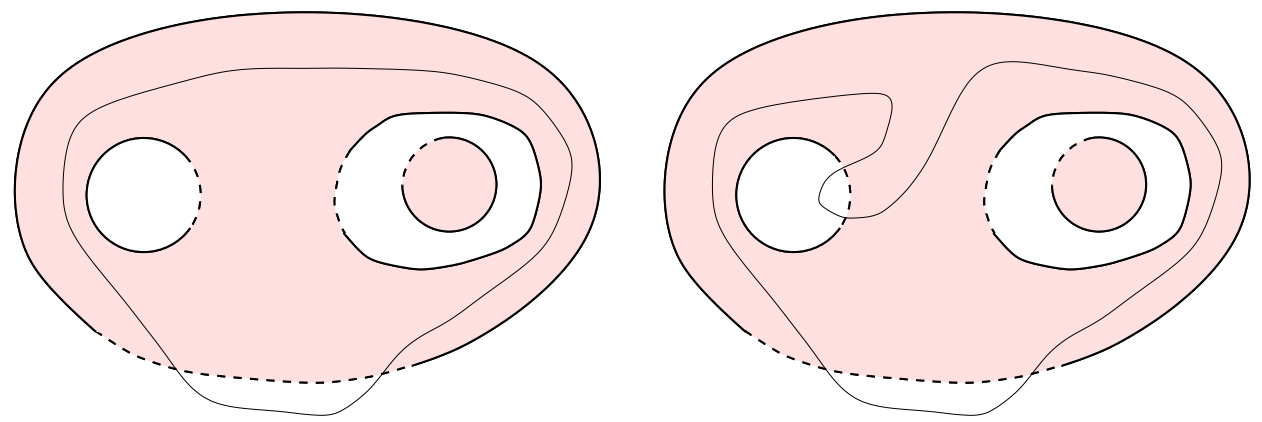

FiguRE 6. After the preceedings steps.
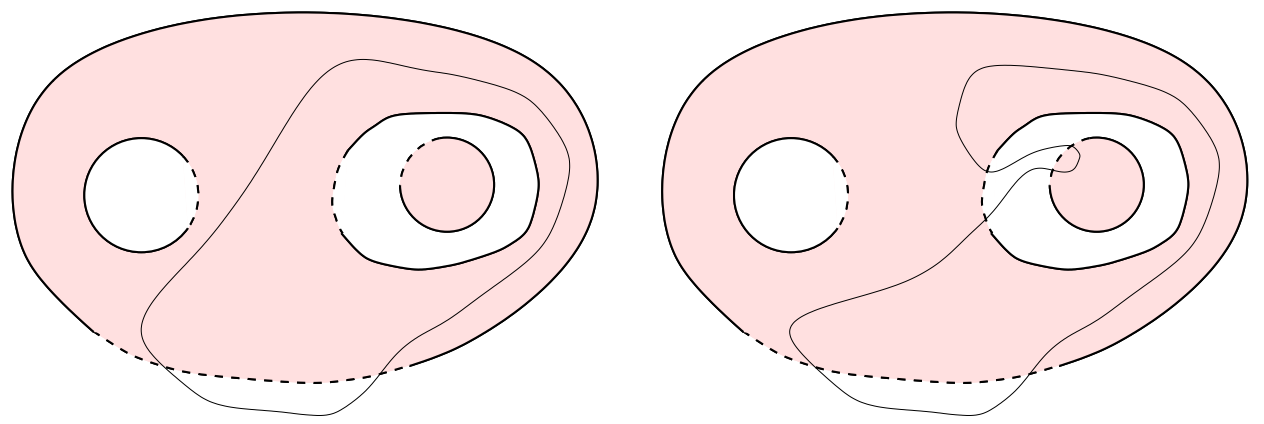

Figure 7 . Once eliminated the first obstacle.
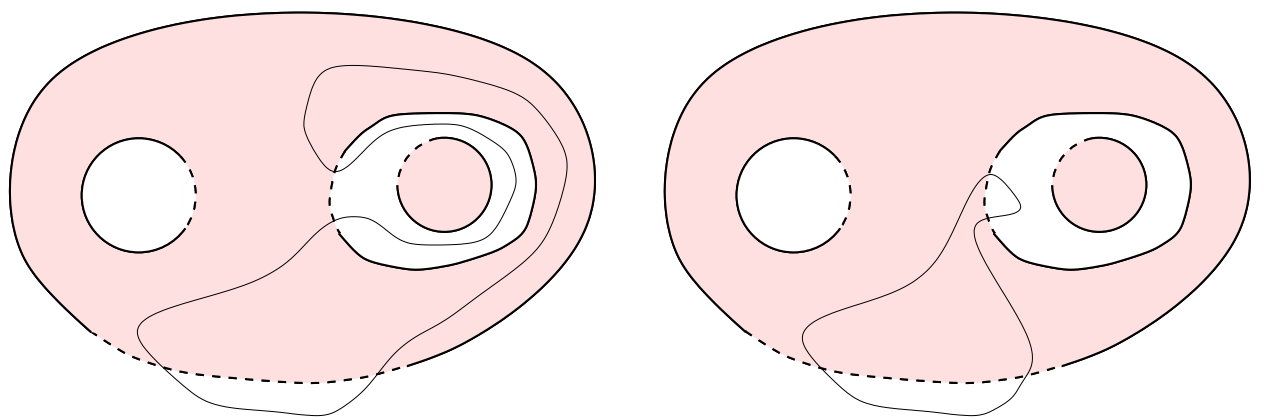

Figure 8. Eliminating the second obstacle. 
The same way as described in these figures, the vortex can "cross" any obstacle in its way in the general case (at each step, one repeats the process described by Figs. 3-5).

Our goal is now to find $\bar{y}^{\mathrm{i}}$ in the form $\nabla \theta(x, t)$ with $\theta$ satisfying $(2.15-2.18)$ and such that the flow of $J_{0}$ along $\bar{y}^{\mathbf{i}}$ is approximately the same as the one along $y^{\mathbf{i}}$.

This is a consequence of the following lemma:

Lemma 7.1. Given $\tilde{J}$ a curve such as described above, and $\mathbf{v} \in C^{\infty}\left(\mathbb{R}^{3} \times[0,1], \mathbb{R}^{3}\right)$, for all $\epsilon>0$, there exists $\theta \in C_{0}^{\infty}\left([0,1] \times \mathbb{R}^{3}, \mathbb{R}^{3}\right)$ satisfying (2.15-2.18), and such that for any $t \in[0,1]$, one has

$$
\left|\phi^{\mathbf{v}}(x, 0, t)-\phi^{\nabla \theta}(x, 0, t)\right|<\epsilon, \forall x \in \tilde{J} .
$$

Moreover, we have the same result on the whole curve $J_{0}$, under the additional assumption that

$$
\int_{\phi^{\mathbf{v}}\left(J_{0}, 0, t\right)} \mathbf{v} \cdot d \tau=0, \forall t \in[0,1] .
$$

We delay the proof of this lemma till the end of the paper. For $\mathbf{v}=y^{i}$, it directly gives us the wanted $\bar{y}^{\mathbf{i}}$, because it suffices then to define $\bar{y}^{\mathbf{i}}:=\nabla \bar{\theta}^{\mathbf{i}}$, where $\bar{\theta}^{\mathbf{i}}$ is given to us by Lemma 7.1. Precisely, we apply the first statement of this lemma for the second stage, and the second one for stages 1 and 3.

Before presenting the exact form of $\aleph^{\mathbf{i}}$, let us make the computation of $\operatorname{curl} \zeta^{\mathbf{i}}(1)$ when for $w^{\mathbf{i}}(0)$ we take a vortex filament along the position of $J$ at the time 0 . That is, we take a linear repartition of Dirac measure on $J_{0}$, say $\mathcal{M}$, and then we set

$$
\bar{w}_{\mathbf{i}}(\cdot, 0)=\mathcal{M} \tau,
$$

where $\tau$ is the unit tangent vector on $J_{0}$ (which sense does not matter much for the moment). Then we will consider a more regular vortex repartition, which will work all the same.

We consider some $f$ as in the statement of Lemma 2.3. Let us prove that, with that choice of $\bar{w}_{\mathbf{i}}(\cdot, 0)$, a solution of (2.22) (in the distribution sense) is given by

$$
\bar{w}_{\mathbf{i}}(\cdot, t)=\mathcal{M}_{\gamma(t)} \tau_{\gamma(t)},
$$

where $\gamma(t)$ is defined as the curve obtained by the flow of $f$ on $J_{0}$ at time $t, \mathcal{M}_{\gamma(t)}$ and $\tau_{\gamma(t)}$ are respectively the linear Dirac repartition and the unit tangent vector on $\gamma(t)$ (also following the flow of $f$ ).

Let us fix a row-vector valued test function $\phi \in C_{0}^{\infty}\left(\tilde{\Omega} \times(0,1), \mathbb{R}^{3}\right)$. Then the function $\bar{w}_{\mathbf{i}}$ described in (7.5) is defined by

$$
\left\langle\bar{w}_{\mathbf{i}}, \phi\right\rangle=\int_{\gamma(t)} \phi(M) d \vec{M}
$$

In other terms, one can write

$$
\left\langle\bar{w}_{\mathbf{i}}, \phi\right\rangle=\int_{[0,1]} \phi\left(\gamma_{t}(v)\right) \gamma_{t}^{\prime}(v) d v .
$$

Let us prove that $\bar{w}_{\mathbf{i}}$ satisfies $(2.22)$. In this part, we denote by a prime the derivative with respect to the variable $v$ and by a point the derivative with respect to the variable $t$. One has

$$
\partial_{t}\left\langle\bar{w}_{\mathbf{i}}, \phi\right\rangle=\int_{[0,1]} \partial_{t}\left(\phi\left(\gamma_{t}(v) \gamma_{t}^{\prime}(v)\right) d v\right.
$$




$$
\partial_{t}\left\langle\bar{w}_{\mathbf{i}}, \phi\right\rangle=\int_{[0,1]} \partial_{t}\left(\phi\left(\gamma_{t}(v)\right)\right) \gamma^{\prime}{ }_{t}(v) d v+\int_{[0,1]} \phi\left(\gamma_{t}(v)\right) \partial_{t}\left(\gamma_{t}{ }_{t}(v)\right) d v
$$

We denote by $Z_{1}$ the first integral, and by $Z_{2}$ the second one. We compute $Z_{1}$ the following way (we put $j$ in upper index for the coordinates)

$$
Z_{1}=\int_{[0,1]}(d \phi)_{\gamma_{t}(v)} \gamma_{t}^{\prime}(v) \dot{\gamma}_{t}(v) d v
$$

Then one has

$$
\begin{aligned}
Z_{1} & =\int_{[0,1]}(d \phi)_{\gamma_{t}(v)}\left(f\left(\gamma_{t}(v)\right)\right) \gamma^{\prime}{ }_{t}(v) d v=\left\langle\bar{w}_{\mathbf{i}}, d \phi_{(\cdot)}(f)\right\rangle=\left\langle\bar{w}_{\mathbf{i}}, \sum_{j} \frac{\partial \phi}{\partial x_{j}} f^{j}\right\rangle=\sum_{j}\left\langle\bar{w}_{\mathbf{i}} f^{j}, \frac{\partial \phi}{\partial x_{j}}\right\rangle \\
& =-\sum_{j}\left\langle\frac{\partial}{\partial x_{j}}\left(\bar{w}_{\mathbf{i}} f^{j}\right), \phi\right\rangle=-\left\langle\sum_{j} f^{j} \frac{\partial}{\partial x_{j}} \bar{w}_{\mathbf{i}}+\bar{w}_{\mathbf{i}} \sum_{j} \frac{\partial}{\partial x_{j}} f^{j}, \phi\right\rangle .
\end{aligned}
$$

So one finally gets

$$
Z_{1}=\left\langle-(f . \nabla) \bar{w}_{\mathbf{i}}-\bar{w}_{\mathbf{i}} \operatorname{div} f, \phi\right\rangle \cdot
$$

We now compute $Z_{2}$ (we denote by $e_{j}$ the $j$-th vector of the canonical basis of $\mathbb{R}^{3}$ )

$$
\begin{aligned}
Z_{2} & =\int_{[0,1]} \phi\left(\gamma_{t}(v)\right)(d f)_{\gamma_{t}(v)}\left(\gamma^{\prime}{ }_{t}(v)\right) d v=\sum_{j, k} \int_{[0,1]} \phi^{k}\left(\gamma_{t}(v)\right) \frac{\partial f^{k}}{\partial x_{j}} \gamma^{\prime j}{ }_{t}(v) d v=\int_{[0,1]} \gamma_{t}^{\prime}(v) \cdot \sum_{j, k} \phi^{k}\left(\gamma_{t}(v)\right) \frac{\partial f^{k}}{\partial x_{j}} e_{j} d v \\
& =\left\langle\bar{w}_{\mathbf{i}}, \sum_{j, k} \phi^{k} \frac{\partial f^{k}}{\partial x_{j}} e_{j}\right\rangle=\sum_{j}\left\langle\bar{w}_{\mathbf{i}}^{j},\left(\sum_{k} \phi^{k} \frac{\partial f^{k}}{\partial x_{j}}\right)\right\rangle=\sum_{j, k}\left\langle\bar{w}_{\mathbf{i}}^{j} \frac{\partial f^{k}}{\partial x_{j}}, \phi^{k}\right\rangle=\left\langle\left(\bar{w}_{\mathbf{i}} \cdot \nabla\right) f, \phi\right\rangle .
\end{aligned}
$$

We easily deduce from $(7.9,7.10)$ and $(7.11)$ that

$\bar{w}_{\mathbf{i}}$ satisfies $(2.22)$ in the distribution sense.

We come back to the proof of (7.1). For that, we compute, using (2.19),

$$
\int_{\Omega} \zeta^{\mathbf{i}}(1) \cdot \mathcal{Q}_{j} d x=\int_{\Omega \times[0,1]} \partial_{t} \zeta^{\mathbf{i}}(t) \cdot \mathcal{Q}_{j} d x d t
$$

The equations $(2.22,2.23)$ and $(2.27)$ imply

$$
\partial_{t} \zeta^{\mathbf{i}}+f \wedge \bar{w}_{\mathbf{i}}=\nabla p,
$$

from which we deduce

$$
\int_{\Omega} \zeta^{\mathbf{i}}(1) \cdot \mathcal{Q}_{j} d x=-\int_{\Omega \times[0,1]} f \wedge \bar{w}_{\mathbf{i}} \cdot \mathcal{Q}_{j} d x d t
$$

Then, we have

$$
\int_{\Omega} \zeta^{\mathbf{i}}(1) \cdot \mathcal{Q}_{j} d x=-\int_{t \in[0,1]} \int_{\tilde{J}(t)} f \wedge \tau \cdot \mathcal{Q}_{j} d \sigma=-\int_{\tilde{\Sigma}_{\mathbf{i}}} \vec{n} \cdot \mathcal{Q}_{j} d \Sigma
$$


where $n$ is the unit normal vector on $\tilde{\Sigma}_{\mathbf{i}}$ with an orientation which depends on the sense chosen for $\omega_{0}^{\mathbf{i}}$ along $J$.

Indeed the function $\sigma:[0,1]^{2} \rightarrow \mathbb{R}^{3}$, defined by $\sigma(s, t):=\gamma(t)(s)$ describes the surface $\tilde{\Sigma}_{\mathbf{i}}$ with elementary area given by

$$
\partial_{t} J \wedge \partial_{s} J=\bar{y}^{\mathbf{i}} \wedge \tau
$$

As $\tilde{\Sigma}_{\mathbf{i}}$ is equivalent to $\Sigma_{\mathbf{i}}$, we have finally the following result

$$
\int_{\Omega} \zeta^{\mathbf{i}}(1) \cdot \mathcal{Q}_{j} d x=-\int_{\Sigma_{\mathbf{i}}} \vec{n} \cdot \mathcal{Q}_{j} d \Sigma
$$

We deduce from the definition of the functions $\mathcal{Q}_{\mathbf{i}}$ that

$$
\int_{\Omega} \mathcal{Q}_{\mathbf{i}} \cdot \mathcal{Q}_{j} d x=\int_{\Omega \backslash \Sigma_{\mathbf{i}}} \nabla q_{\mathbf{i}} \cdot \nabla q_{j} d x=-\int_{\Sigma_{\mathbf{i}}}\left(\mathcal{Q}_{j} \cdot n\right) d \Sigma
$$

Therefore, we get

$$
\int_{\Omega} \zeta^{\mathbf{i}}(1) \cdot \mathcal{Q}_{j} d x=\int_{\Omega} \mathcal{Q}_{\mathbf{i}} \cdot \mathcal{Q}_{j} d x
$$

As by construction of $\bar{y}^{\mathbf{i}}$ and $\bar{w}_{\mathbf{i}}$,

$$
\operatorname{curl} \zeta^{\mathbf{i}}(1)=0
$$

the previous equality implies

$$
\zeta^{\mathbf{i}}(1)=\mathcal{Q}_{\mathbf{i}}
$$

Now we want to have an equivalent result, but with a more regular vortex repartition. Instead of (7.4), we take here, for $\epsilon<1$,

$$
w^{\mathbf{i}}(\cdot, 0)=\mathcal{M} * \rho_{\epsilon} \tau
$$

where $\rho_{\epsilon}$ is a regularisation kernel with support in the ball with center 0 and radius $\epsilon$ in $\mathbb{R}^{3}$, positive and which moreover satisfies

$$
\int_{\mathbb{R}^{3}} \rho_{\epsilon} d x=1
$$

Let us take in (7.17) $\epsilon$ small enough, in such a way that, if we consider the solution of (2.22), then one has

$$
\text { Supp } w^{\mathbf{i}}(\cdot, 1) \subset \tilde{\Omega} \backslash \bar{\Omega} .
$$

Then by (7.12) we get as a solution of $(2.22)$

$$
w^{\mathbf{i}}(\cdot, t)=\int_{B(0, \epsilon)} \mathcal{M}_{\phi^{f}(0, t,-x+\gamma)} \tau_{\phi^{f}(0, t,-x+\gamma)} \rho_{\epsilon}(x) d x .
$$

In particular, one gets that $w^{\mathbf{i}}$ is solenoidal. Moreover, using the previous calculus on the linear repartition and (7.18), one deduces that we still get the result that for any $x \in B(0, \epsilon)$

$$
\int_{t \in[0,1]} \mathcal{Q}_{j} . f \wedge \mathcal{M}_{\phi^{f}(0, t,-x+\gamma)} \tau_{\phi^{f}(0, t,-x+\gamma)}=-\int_{\Omega} \mathcal{Q}_{i} \cdot \mathcal{Q}_{j}
$$


so after integration in variable $x$, with (7.18), one gets the same way as previously that

$$
\int_{t \in[0,1]} f \wedge w^{i}=-\int_{\Omega} \mathcal{Q}_{i} \cdot \mathcal{Q}_{j}
$$

which leads all the same to

$$
\zeta^{\mathbf{i}}(1)=\mathcal{Q}_{\mathbf{i}},
$$

which completes the proof.

\section{Proof of Lemma 6.2}

We argue by contradiction. If this proposition was not true, then for some $\bar{x} \in \partial \Omega$, there would exist a vector $V \in T_{\bar{x}}(\partial \Omega) \backslash\{0\}$, such that for any $\phi$ in $C^{\infty}(\bar{\Omega} ; \mathbb{R})$, with

$$
\Delta \phi=0 \text { in } \bar{\Omega}, \quad \frac{\partial \phi}{\partial n}=0 \text { on } \Gamma_{0},
$$

stands

$$
V . \nabla \phi(\bar{x})=0 .
$$

In particular, for $\Omega^{\#}$ as in Figure 1, we will consider functions $\phi^{\bar{a}, a}$ defined for $a, \bar{a} \in \Omega^{\#}$ by

$$
\left\{\begin{array}{l}
\Delta \phi^{\bar{a}, a}=4 \pi\left(\delta_{a}-\delta_{\bar{a}}\right) \text { in } \Omega \cup \Omega^{\#}, \\
\partial_{n} \phi^{\bar{a}, a}=0 \text { in } \partial\left(\overline{\Omega \cup \Omega^{\#}}\right), \\
\int_{\Omega} \phi^{\bar{a}, a}=0 .
\end{array}\right.
$$

Because of the analyticity in $a \in \overline{\Omega \cup \Omega^{\#}} \backslash \partial\left(\Omega \cup \Omega^{\#}\right)$ of $V \cdot \nabla \phi^{\bar{a}, a}(\bar{x})$, the relation

$$
V \cdot \nabla \phi^{\bar{a}, a}(\bar{x})=0,
$$

which holds for $a \in \Omega^{\#}$, remains true when $a \in \Omega$.

Let $\bar{a}$ be constant in $\Omega^{\#}$, so we will note simply $\phi^{a}:=\phi^{a, \bar{a}}$. We want to prove that (8.2) is false, by making $a$ approach $\bar{x}$.

Let us denote by $\mathfrak{S}$ the orthogonal symmetry with respect to $T_{\bar{x}}(\partial \Omega)$.

We now consider the functions $\hat{\phi}^{a}$ defined by

$$
\hat{\phi}^{a}(x)=\frac{1}{|x-a|}+\frac{1}{|x-\mathfrak{S}(a)|}-\frac{1}{|x-\bar{a}|} \text { in } \mathbb{R}^{3} .
$$

This corresponds to the 3D-potential created by 3 particles placed in $a, \mathfrak{S}(a)$ and $\bar{a}$, with respective charge 1 , 1 and -1 . We also have

$$
\Delta \hat{\phi}^{a}=4 \pi\left(\delta_{a}+\delta_{\mathfrak{S}(a)}-\delta_{\bar{a}}\right) \text { in } \mathbb{R}^{3} .
$$

Let $N(a):=|a-\bar{x}|^{2}$. We normalize our functions $\phi^{a}$ and $\hat{\phi}^{a}$ by setting

$$
\left\{\begin{array}{l}
\psi^{a}:=N(a) \phi^{a} / 2 \\
\hat{\psi}^{a}:=N(a) \hat{\phi}^{a} / 2
\end{array}\right.
$$


We now want to prove that for $a$ "close" to $\bar{x}, \nabla \psi^{a}(\bar{x})$ is "close"' to $\nabla \hat{\psi}^{a}(\bar{x})$, and that, if wanted, $\nabla \hat{\psi}^{a}(\bar{x}) . V$ is close to $\|V\|$.

This last point is easy to see. Indeed,

$$
\nabla \hat{\psi}^{a}(x)=N(a)\left(\frac{a-x}{|x-a|^{3}}+\frac{\mathfrak{S}(a)-x}{|x-\mathfrak{S}(a)|^{3}}-\frac{\bar{a}-x}{|x-\bar{a}|^{3}}\right) \text { in } \mathbb{R}^{3},
$$

so at the point $\bar{x}$ we get, as $a \rightarrow \bar{x}$,

$$
\nabla \hat{\psi}^{a}(\bar{x})=\frac{\bar{x}-P(a)}{|\bar{x}-a|}+o(1)
$$

where $P$ is the orthogonal projector on $T_{\bar{x}}(\partial \Omega)$. So we can approach by $\nabla \hat{\psi}^{a}(\bar{x})$ any unit vector of the tangent plane, in particular $\frac{V}{\|V\|}$.

In the rest of the proof, $a$ will converge to $\bar{x}$ along straight lines passing through $\bar{x}$ and non tangent to $\partial \Omega$ at point $\bar{x}$, in such a way that

$$
d(a, \partial \Omega) \geq c d(a, \bar{x}),
$$

when $a \rightarrow \bar{x}$, for a certain $c>0$, which depends on the chosen direction.

Now to prove Lemma 6.2 , we have left to prove that $\nabla \psi^{a}(\bar{x})$ is "close"' to $\nabla \hat{\psi}^{a}(\bar{x})$.

This property relies on the fact that $\partial_{n} \hat{\psi}^{a}$ is small for $a$ close to $\bar{x}$ in an Hölder norm $C^{0, \alpha}$ with $\alpha$ small enough (for example $\alpha<\frac{1}{6}$ ).

Indeed, we clearly have

$$
\Delta\left(\phi^{a}-\hat{\phi}^{a}\right)=0 \text { in } \tilde{\Omega} .
$$

Hence we can bound $\left(\phi^{a}-\hat{\phi}^{a}\right)$ the following way, using an elliptic regularity property with $C^{0, \alpha}$ Neumann boundary condition (for which we refer to $[8]$ ):

$$
\left\|\nabla \psi^{a}-\nabla \hat{\psi}^{a}\right\|_{C^{0, \alpha}(\Omega)} \leq C\left\|\partial_{n} \hat{\psi}^{a}\right\|_{C^{0, \alpha}(\partial \Omega)},
$$

and consequently

$$
\left\|\nabla \psi^{a}-\nabla \hat{\psi}^{a}\right\|_{C^{0, \alpha}(\partial \Omega)} \leq C\left\|\partial_{n} \hat{\psi}^{a}\right\|_{C^{0, \alpha}(\partial \Omega)} .
$$

Let us now prove that

$$
\partial_{n} \hat{\psi}^{a} \stackrel{C^{0, \alpha}(\partial \Omega)}{\longrightarrow} 0
$$

as $a \rightarrow \bar{x}$.

We denote by $u^{a}(x)$ the unit vector $(x-a) /|x-a|$ and by $\rho^{a}(x)$ the scalar function $\left(1 /|x-a|^{2}\right)$. Hence, we have

$$
\partial_{n} \hat{\phi}^{a}(x)=\left(\rho^{a}(x) u^{a}(x)+\rho^{\mathfrak{S}(a)}(x) u^{\mathfrak{S}(a)}(x)\right) \cdot n(x) \text { on } \partial \Omega .
$$

From the previous equation, we deduce:

$$
\begin{aligned}
\left\|\partial_{n} \hat{\phi}^{a}\right\|_{0, \alpha, X} & \leq\left\|\rho^{a}\right\|_{0, \alpha, X} \cdot\left\|\left(u^{a} \cdot n\right)\right\|_{0, X}+\left\|\rho^{a}\right\|_{0, X} \cdot\left\|\left(u^{a} \cdot n\right)\right\|_{0, \alpha, X}+\left\|\rho^{\mathfrak{S}(a)}\right\|_{0, \alpha, X} \cdot\left\|\left(u^{\mathfrak{S}(a)} \cdot n\right)\right\|_{0, X} \\
& +\left\|\rho^{\mathfrak{S}(a)}\right\|_{0, X} \cdot\left\|\left(u^{\mathfrak{S}(a)} \cdot n\right)\right\|_{0, \alpha, X},
\end{aligned}
$$


for any open part $X$ of the boundary. Equation (8.10) will be useful for points far from $\bar{x}$.

We consequently estimate $\left\|\rho^{a}\right\|_{0, X}$ and $\left\|\rho^{a}\right\|_{0, \alpha, X}$.

It is quite clear that

$$
\left\|\rho^{a}\right\|_{0, X} \leq C \frac{1}{d^{2}(a, X)}
$$

We now estimate $\left\|\rho^{a}\right\|_{0, \alpha, X}$ and $\left\|\rho^{\mathfrak{S}(a)}\right\|_{0, \alpha, X}$. It is clear that it is sufficient to estimate the first norm, because the same estimate will hold also for the second one.

We introduce the functions, defined for any $x \in X$ (in fact for any $x$ such that $d(x, a) \geq d(X, a)$ ) as follows

$$
\mathfrak{U}_{y}^{x}=\frac{\rho^{a}(x)-\rho^{a}(y)}{|x-y|^{\alpha}}, \quad \forall y \neq x .
$$

Let $d:=d(X, a)$. We now want to estimate $\mathfrak{U}_{y}^{x}$. For $x$ with $d(x, a) \geq d(X, a)$, the value

$$
\max _{\{y /|x-y|=h, d(y, a) \geq d / 2\}}\left(\frac{1}{|x-a|^{2}}-\frac{1}{|y-a|^{2}}\right)
$$

is less or equal than the maximum of the value obtained for

$$
y=x-d / 2(x-a) /|x-a|
$$

(for $h>d / 2$ ), and of the one obtained for

$$
y=x-h(x-a) /|x-a|
$$

(for $h \leq d / 2$ ). Consequently, we now have left to estimate

$$
m_{1}=\max _{0<h<d / 2} h^{-\alpha}\left(\frac{1}{|x-a|^{2}}-\frac{1}{|x-h((x-a) /|x-a|)-a|^{2}}\right),
$$

and

$$
m_{2}=\max _{h>d / 2} h^{-\alpha}\left(\frac{1}{|x-a|^{2}}-\frac{1}{|x-d / 2((x-a) /|x-a|)-a|^{2}}\right)
$$

A simple study of these numerical functions shows that these maximum are obtained in the first case for $h$ satisfying the equation

$$
-\alpha|1-h|^{3}+\alpha|1-h|-h=0,
$$

which is a value independent from the choice of $x$ in the suitable set. In the second case, one obtains that the maximum is obtained for $h=d / 2$.

As a consequence, we can deduce an inequality (with $C$ a constant independent of $x$ )

$$
\max _{y}\left(\frac{1}{|x-a|^{2}}-\frac{1}{|y-a|^{2}}\right) /\left(|x-y|^{\alpha}\right) \leq C(d(X, a))^{-2-\alpha} .
$$

Finally, we can deduce from the previous inequalities that

$$
\left\|\rho^{a}\right\|_{0, \alpha, \partial \Omega} \leq C[d(a, \partial \Omega)]^{-2-\alpha} .
$$


We consider next $\left\|u^{a}\right\|_{0, X}$ and $\left\|u^{a}\right\|_{0, \alpha, X}$. Of course $\left\|u^{a}\right\|_{0, X} \leq 1$.

We now consider $\left\|u^{a}\right\|_{0, \alpha, X}$. As $u^{a}(x)$ is constant along the half-lines with origin $a$, it is obvious that the greatest values of the quotient

$$
\frac{\left|u^{a}(x)-u^{a}(y)\right|}{|x-y|^{\alpha}}
$$

for $x \neq y$ and $\min (d(a, x), d(a, y)) \leq d$, are obtained when $d(a, x)=d(a, y)=d$.

By using polar coordinates with center $a$, we can see also that for some $C>0$ independent from $a$,

$$
\left\|u^{a}\right\|_{0, \alpha, X} \leq C \frac{1}{d(X, a)^{\alpha}}
$$

which is in fact still valid when $\alpha=1$.

We now deal with $\left\|\nabla \psi^{a} . n\right\|_{0, X}$ and $\left\|\nabla \psi^{a} . n\right\|_{0, \alpha, X}$ directly. The estimate we get here will be used for points near $\bar{x}$. Of course, $\left\|\left(\nabla \psi^{a} . n\right)\right\|_{0, X} \leq 1$, and the norm $\left\|\left(\nabla \psi^{a} . n\right)\right\|_{0, \alpha, X}$ will get our whole attention.

Here, for $d>0$, we introduce $X^{d}:=\partial \Omega \cap B(\bar{x}, d)$. Let us show the next formula, for a certain $C>0$ independent from $a$ and $d$ :

$$
\left\|\nabla \psi^{a} . n\right\|_{0, \alpha, X^{d}} \leq C\left(d^{1-\alpha}+\frac{d^{3-\alpha}}{|\bar{x}-a|^{2}}\right)
$$

For $x, y \in X^{d}$ with $x \neq y$, we set:

$$
I_{x, y}=\left(\nabla \psi^{a}(y) \cdot n(y)-\nabla \psi^{a}(x) \cdot n(x)\right)|x-y|^{-\alpha},
$$

and we have

$$
\begin{aligned}
I_{x, y}=\nabla \psi^{a}(x) \cdot(n(y)-n(x))|x-y|^{-\alpha} & +(n(y)-n(\bar{x}))\left(\nabla \psi^{a}(y)-\nabla \psi^{a}(x)\right)|x-y|^{-\alpha} \\
& +n(\bar{x}) \cdot\left(\nabla \psi^{a}(y)-\nabla \psi^{a}(x)\right)|x-y|^{-\alpha} .
\end{aligned}
$$

We evaluate the first term. Clearly,

$$
\left|\nabla \psi^{a}(x) \cdot(n(y)-n(x))\right| x-\left.y\right|^{-\alpha}|\leq| \nabla \psi^{a}(x)\left|\|n\|_{1, \partial \Omega}\right| x-\left.y\right|^{1-\alpha} \leq C d^{1-\alpha} .
$$

We then evaluate the second one. We compute

$$
\left|(n(y)-n(\bar{x}))\left(\nabla \psi^{a}(y)-\nabla \psi^{a}(x)\right)\right| x-\left.y\right|^{-\alpha}|\leq C(\Omega)| \bar{x}-y\left|\left\|\nabla \psi^{a}\right\|_{1, X^{d}}\right| x-\left.y\right|^{1-\alpha} .
$$

Let us interest ourselves to the term " $\left\|\nabla \psi^{a}\right\|_{1, X^{d}}$ ". From (8.3) and (8.5), one deduces that for some $C>0$, one has

$$
\left\|\nabla \psi^{a}\right\|_{1, X^{d}} \leq \frac{C}{|a-\bar{x}|}
$$

Hence, taking (8.8) in account, we get

$$
\left|(n(y)-n(\bar{x}))\left(\nabla \psi^{a}(y)-\nabla \psi^{a}(x)\right)\right| x-\left.y\right|^{-\alpha} \mid \leq C(\Omega, c) \frac{d^{2-\alpha}}{|a-\bar{x}|} .
$$


We finally evaluate the third term in (8.16). One has

$$
\left|n(\bar{x}) \cdot\left(\nabla \psi^{a}(x)-\nabla \psi^{a}(y)\right)\right| x-\left.y\right|^{-\alpha}\left|\leq\left\|\nabla \psi^{a}(\cdot) \cdot n(\bar{x})\right\|_{C^{1}\left(X^{d}\right)}\right| x-\left.y\right|^{1-\alpha}
$$

We remark that $\nabla \psi^{a}(x) \cdot n(\bar{x})=0$ for any $x$ in $T_{\bar{x}} \partial \Omega$. Then

$$
\left\|\nabla \psi^{a}(\cdot) . n(\bar{x})\right\|_{C^{1}\left(X^{d}\right)} \leq\left\|\nabla \psi^{a} . n(\bar{x})\right\|_{2, X^{d}} d\left(X^{d}, T_{\bar{x}} \partial \Omega\right) \leq\left\|\nabla \psi^{a} . n(\bar{x})\right\|_{2, X^{d}} d^{2} \leq C \frac{d^{2}}{|a-\bar{x}|^{2}} .
$$

So one has

$$
\left|n(\bar{x}) \cdot\left(\nabla \psi^{a}(x)-\nabla \psi^{a}(y)\right)\right| x-\left.y\right|^{-\alpha} \mid \leq C \frac{d^{3-\alpha}}{|a-\bar{x}|^{2}}
$$

Putting together estimates $(8.17,8.18)$ and $(8.19)$, we get $(8.15)$.

Let us now prove (8.9). We consider for $\epsilon>0$

$$
\beta:=\frac{2+\epsilon}{3-\alpha}
$$

We choose $\epsilon$ small enough (for example $\epsilon<\frac{1}{6}$ ) such that one has

$$
2-\beta(2+\alpha)>0
$$

We distinguish the points of $\partial \Omega$, located inside and outside the open ball $B\left(a,|a-\bar{x}|^{\beta}\right)$ (which obviously meets $\partial \Omega$ for $N(a)<1)$.

If $x \notin B\left(a, N(a)^{\frac{\beta}{2}}\right)$, then we have $|x-a| \geq|a-\bar{x}|^{\beta}$ and consequently, with $(8.6,8.10,8.13)$ and (8.14), we get

$$
\left\|\partial_{n} \hat{\psi}^{a}\right\|_{0, \alpha,\left[\partial \Omega \backslash B\left(a, N(a)^{\frac{\beta}{2}}\right)\right]} \leq C|\bar{x}-a|^{2-\beta(2+\alpha)} .
$$

We have to treat the case when $x \in B\left(a, N(a)^{\frac{\beta}{2}}\right)$. We omit the term concerning $\bar{a}$ in $(8.6)$, which obviously has no importance to estimate $\left\|\partial_{n} \hat{\psi}^{a}\right\|$. We use (8.15) and get, with (8.20),

$$
\left\|\partial_{n} \hat{\psi}^{a}\right\|_{0, \alpha,\left[\partial \Omega \cap B\left(a, N(a)^{\frac{\beta}{2}}\right)\right]} \leq C\left(|a-\bar{x}|^{\beta(1-\alpha)}+|a-\bar{x}|^{\epsilon}\right)
$$

Finally, we conclude that $\left\|\partial_{n} \hat{\psi}^{a}\right\|_{C^{0, \alpha}}$ tends to 0 and that ends the proof.

\section{Proof of Lemma 7.1}

\subsection{Preliminary Lemmas}

The main purpose of section 9 is to prove the first statement of Lemma 7.1, that is the one relative to $\tilde{J}$. At the end of the section, we will come back to the case when we wish to have a control on the whole curve $J_{0}$.

We first establish a lemma which corresponds to the control of paths inside $\Omega$. Precisely, we prove 
Lemma 9.1. Let $\gamma:[0, L] \rightarrow \bar{\Omega}$ be a smooth path, injective, with $\gamma((0, L)) \subset \Omega$, and $\gamma(0), \gamma(L) \in \partial \Omega$, and such that for any $s$ in $[0, L],|\dot{\gamma}(s)|=1$. Suppose also that $\dot{\gamma}(s) \notin T_{\gamma(s)}(\partial \Omega)$ for $s=0, L$.

Let $y \in C^{\infty}\left(\gamma, \mathbb{R}^{3}\right)$ be a vector field defined on $\gamma$ such that

$$
y(\gamma(0)) \cdot n(\gamma(0))=y(\gamma(L)) \cdot n(\gamma(L))=0 .
$$

Then for any $\epsilon>0$, there exists $\theta \in C_{0}^{\infty}\left(\mathbb{R}^{3}, \mathbb{R}\right)$ such that the following properties hold:

$$
\begin{gathered}
\Delta \theta=0 \text { in } \Omega, \\
\partial_{n} \theta=0 \text { on } \partial \Omega \backslash \Gamma_{0}, \\
\|\nabla \theta-y\|_{C^{0}(\gamma)}<\epsilon .
\end{gathered}
$$

Actually, we need

Corollary 9.2. Given $\gamma:[0, L] \rightarrow \bar{\Omega}$ a smooth path, injective, satisfying $\gamma((0, L)) \subset \Omega$, and $\gamma(0), \gamma(L) \in \partial \Omega$, and given $\epsilon>0$, there exists $\delta(\gamma, \epsilon)>0$, and $\mathcal{W}(\gamma, \epsilon)>0$, such that for any $\tilde{\gamma}$ such that $\|\gamma-\tilde{\gamma}\|_{C^{0}}<\delta$, for any $\tilde{y}$ in $C^{1}\left(\gamma ; \mathbb{R}^{3}\right)$ satisfying (9.1), one can find $\theta$ such that (9.2) and (9.3) hold, and such that

$$
\begin{gathered}
\|\tilde{y} \circ \tilde{\gamma}-\nabla \theta \circ \tilde{\gamma}\|_{C^{0}([0, L])}<\epsilon\|\tilde{y}\|_{C^{0}([0, L]) ; \mathbb{R}^{3}}, \\
\|\theta\|_{C^{2}(\Omega)} \leq \mathcal{W}\|\tilde{y}\|_{C^{1}\left(\gamma ; \mathbb{R}^{3}\right)} .
\end{gathered}
$$

\subsection{Proof of Lemma 9.1}

Let us denote $\Gamma:=\gamma([0, L])$. We first remark that the map which associates to $\theta$ satisfying (9.2) and (9.3), the function $\left(\nabla \theta_{\mid \Gamma}\right) \in C^{0}(\Gamma)$ is linear.

We argue by contradiction and suppose that there exists a non-zero measure $M \in \mathcal{M}\left(\Gamma, \mathbb{R}^{3}\right)$ (viz. the Radon measures defined on $\Gamma$ ), precisely belonging to the set

$$
\left\{y \in C^{0}\left(\Gamma, \mathbb{R}^{3}\right) / y(\gamma(0)) \cdot n(\gamma(0))=y(\gamma(L)) \cdot n(\gamma(L))=0\right\}^{\prime}=\frac{\mathcal{M}\left(\Gamma, \mathbb{R}^{3}\right)}{\operatorname{Vect}\left\{\delta_{\gamma(0)} \cdot n(\gamma(0)), \delta_{\gamma(1)} \cdot n(\gamma(L))\right\}},
$$

such that:

$$
\langle M, \nabla \theta\rangle_{\mathcal{M}(\Gamma) \times C^{0}(\Gamma)}=0,
$$

for all $\theta \in C^{\infty}(\Omega, \mathbb{R})$ satisfying $(9.2)$ and (9.3).

To find a contradiction, we introduce, as for Lemma 2.1, an "over-domain" $\Omega^{\#}$ described by figure 1 , and the functions $\phi^{\bar{a}, a}$ defined on $\Omega^{\#}$ by

$$
\left\{\begin{array}{l}
\Delta \phi^{a, \bar{a}}=4 \pi\left(\delta_{a}-\delta_{\bar{a}}\right) \text { in } \Omega^{\#} \\
\partial_{n} \phi^{a, \bar{a}}=0 \text { on } \partial \Omega^{\#} \\
\int_{\Omega^{\#}} \phi^{a, \bar{a}}=0
\end{array}\right.
$$

As in Lemma 6.2, relation (9.8), which is true for $\theta=\phi^{a, \bar{a}}$ when $a$ is in $\Omega^{\#} \backslash \bar{\Omega}$, is still valid when $a$ is in $\Omega$, as a consequence of the analyticity of $\left\langle M, \nabla \phi^{\bar{a}, a}\right\rangle_{\mathcal{M}(\Gamma) \times C^{0}(\Gamma)}$ in variable $a$. 
In the rest of the proof, we will omit $\bar{a}$ whose corresponding term has not importance, and generally, instead of dealing with the function $\phi^{a, \bar{a}}$, we will work on the function

$$
x \mapsto \frac{1}{|x-a|},
$$

which is close to $\phi^{a, \bar{a}}$, for $x$ close to $a$ in $\Omega$. This can be done because effects of $\bar{a}$ and of the Neumann condition on the boundary are negligeable.

In a first step, we prove that the measure $M$ is necessarily tangent on $\Gamma$.

Let $\tau$ be defined on $\Gamma$ by $\tau(\gamma(s)):=\frac{d}{d s} \gamma(s)$. We also introduce a $C^{\infty}$ function $\nu(x)$ of unit normal vectors defined on $\Gamma$. We consider a fixed point $x_{0}:=\gamma\left(s_{0}\right)$ of $\Gamma \backslash\{\gamma(0), \gamma(1)\}$ and for any $\lambda \in \mathbb{R}$ the point $a\left(x_{0}, \lambda\right):=$ $x_{0}-\lambda \nu\left(x_{0}\right)$ (placed as described in Fig. 9), which we will denote by $a(\lambda)$ or even $a$ when there is no possible ambiguity. We use as a parameterization of $\Gamma$ the arc length from $x_{0}$, which we denote again by $s$.

First, let us prove that, for all vectorial continuous function $f$ defined on $\Gamma$, one has

$$
\lambda \int_{\Gamma} f(x) \cdot \nabla \phi^{a\left(x_{0}, \lambda\right)} d \tau \longrightarrow 2 f\left(x_{0}\right) \cdot \nu\left(x_{0}\right), \text { as } \lambda \rightarrow 0
$$

In that purpose, we introduce a new function $\eta$, defined once $x_{0}$ is fixed, and depending on the variables $s$ and

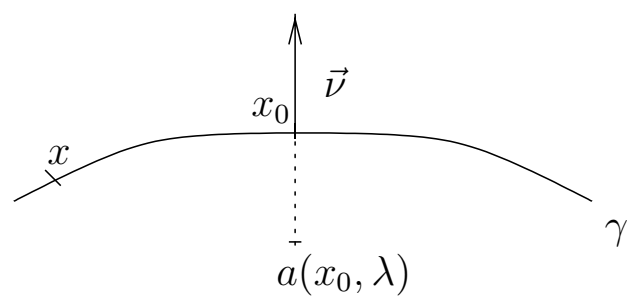

Figure 9. The position of the particle approaching $x_{0}$.

$\lambda$. It is indeed quite easy to see that:

$$
|a(\lambda)-\gamma(s)|^{2}=\left|a(\lambda)-x_{0}\right|^{2}+s^{2} \eta(s, \lambda),
$$

where $\eta$ is a continuous uniformly bounded function defined for $s$ in a certain interval $\left[-\alpha_{0}, \alpha_{0}\right]$ and for $\lambda$ in $[0, \hat{\lambda}]$. Moreover, there exists some $m>0$ such that, for $\alpha$ small enough, one has

$$
|\eta(s, a)| \geq m \text { on } \gamma_{\alpha}, \text { for all } \lambda \in[0, \hat{\lambda}]
$$

We remark that $\eta(0, \lambda)=1$ for all $\lambda \in[0, \hat{\lambda}]$.

For $\alpha>0$, let us denote by $\Gamma_{\alpha}$ the arc $\gamma\left(\left[s_{0}-\alpha, s_{0}+\alpha\right]\right)$.

We fix $\epsilon>0$. We deduce from the continuity of the function $(s, \lambda) \mapsto f(s) \cdot\left(\gamma(s)-a\left(x_{0}, \lambda\right)\right)$, that, for a certain $\lambda_{0}>0$, and a certain $\alpha>0$ one has

$$
\left|[(\gamma(s)-a(\lambda)) \cdot f(\gamma(s))]-\left[\left(x_{0}-a(\lambda)\right) . f\left(x_{0}\right)\right]\right|<\epsilon, \forall s \in[-\alpha, \alpha], \forall \lambda<\lambda_{0}
$$

In this expression, we remark that $\left(x_{0}-a\right) \cdot f\left(x_{0}\right)=\lambda(a)(f . \nu)\left(x_{0}\right)$.

We introduce the function $\Theta_{\lambda}:=\lambda \phi^{a\left(x_{0}, \lambda\right)}$, so that we are interested in the limit of

$$
\int_{\Gamma} f(x) \cdot \nabla \Theta_{\lambda} d \tau
$$


when $\lambda \rightarrow 0$. We cut this integral the following way:

$$
\int_{\Gamma} f . \nabla \Theta_{\lambda} d \sigma=\int_{\Gamma_{\alpha}} f . \nabla \Theta_{\lambda} d \sigma+\int_{\Gamma \backslash \Gamma_{\alpha}} f . \nabla \Theta_{\lambda} d \sigma .
$$

Let us prove the second of these two integrals tends to zero as $\lambda \rightarrow 0$. Indeed, we know that on $\Gamma \backslash \Gamma_{\alpha}$

$$
\nabla \phi^{a(\lambda)}(x) \longrightarrow \nabla \phi^{x_{0}}(x)
$$

as $\lambda \rightarrow 0$, uniformly in $x$. So, considering integrals, we get that

$$
\int_{\Gamma \backslash \Gamma_{\alpha}} \nabla \phi^{a(\lambda)} d \sigma \text { is bounded, }
$$

and hence multiplying it by $\lambda$, we obtain an integral which converges to 0 as $\lambda \rightarrow 0$.

We are now interested in the first integral in the right hand side of (9.14), which we call $I_{1}$. First, as $x_{0} \notin \partial \Omega$, the difference between the two functions

$$
\nabla \phi^{a(\lambda)} \text { and } x \mapsto \frac{x-a}{|x-a|^{3}},
$$

is bounded as $\lambda$ tends to 0 . So considering integrals and multiplying by $\lambda$, we get the existence of $\lambda_{1}<\lambda_{0}$ such that, if we set

$$
I_{2}:=\int_{\Gamma_{\alpha}} \frac{\lambda(x-a(\lambda)) \cdot f}{|x-a(\lambda)|^{3}} d \sigma
$$

then one has, for $\lambda<\lambda_{1}$,

$$
\left|I_{1}-I_{2}\right|<\epsilon
$$

Using (9.10) and (9.12), we get

$$
\left|I_{2}-\int_{-\alpha}^{\alpha} \frac{(f . \nu)\left(x_{0}\right) \lambda^{2}}{\left(\lambda^{2}+s^{2} \eta(a, s)\right)^{\frac{3}{2}}} d s\right| \leq \epsilon \int_{-\alpha}^{\alpha} \frac{\lambda^{2}}{\left(\lambda^{2}+s^{2} \eta(a, s)\right)^{\frac{3}{2}}} d s,
$$

and then,

$$
\left|I_{2}-\int_{-\alpha}^{\alpha} \frac{(f . \nu)\left(x_{0}\right)}{\left(1+\left(\frac{s}{\lambda}\right)^{2} \eta(a, s)\right)^{\frac{3}{2}}} d\left(\frac{s}{\lambda}\right)\right| \leq \epsilon \int_{-\alpha}^{\alpha} \frac{1}{\left(1+\left(\frac{s}{\lambda}\right)^{2} \eta(a, s)\right)^{\frac{3}{2}}} d\left(\frac{s}{\lambda}\right) .
$$

Now we use (9.11) and consequently get

$$
\left|I_{2}-\int_{-\alpha}^{\alpha} \frac{(f . \nu)\left(x_{0}\right)}{\left(1+\left(\frac{s}{\lambda}\right)^{2} \eta(a, s)\right)^{\frac{3}{2}}} d\left(\frac{s}{\lambda}\right)\right| \leq \epsilon \int_{-\infty}^{\infty} \frac{1}{\left(1+m t^{2}\right)^{\frac{3}{2}}} d t .
$$

We introduce the new notation

$$
I_{3}:=\int_{-\alpha}^{\alpha} \frac{(f \cdot \nu)\left(x_{0}\right)}{\left(1+\left(\frac{s}{\lambda}\right)^{2} \eta(a, s)\right)^{\frac{3}{2}}} d\left(\frac{s}{\lambda}\right) .
$$


We now want to know the limit of $I_{3}$. We compute

$$
I_{3}=\int_{-\frac{\alpha}{\lambda}}^{\frac{\alpha}{\lambda}} \frac{(f . \nu)\left(x_{0}\right)}{\left(1+t^{2} \eta(a, \lambda t)\right)^{\frac{3}{2}}} d t .
$$

We extend $\eta$ to $\mathbb{R}$ by making it equal to $\eta\left(-\alpha_{0}\right)$ on $\left(-\infty,-\alpha_{0}\right]$ and to $\eta\left(\alpha_{0}\right)$ on $\left[\alpha_{0},+\infty\right)$, for any $\lambda$. Then, we remark that

$$
I_{4}:=\int_{-\infty}^{\infty} \frac{(f . \nu)\left(x_{0}\right) d t}{\left(1+t^{2} \eta(a, \lambda t)\right)^{\frac{3}{2}}} \leq \int_{-\infty}^{\infty} \frac{\left|(f . \nu)\left(x_{0}\right)\right| d t}{\left(1+m t^{2}\right)^{\frac{3}{2}}}<+\infty .
$$

As the following inequality stands

$$
\left|I_{4}-I_{3}\right| \leq \int_{\mathbb{R} \backslash\left(-\frac{\alpha}{\lambda}, \frac{\alpha}{\lambda}\right)} \frac{(f \cdot \nu)\left(x_{0}\right) d t}{\left(1+t^{2} m\right)^{\frac{3}{2}}},
$$

the difference $\left|I_{4}-I_{3}\right|$ goes to 0 when $\lambda \rightarrow 0$.

We have now to search for the limit of the integral $I_{4}$ as $\lambda \rightarrow 0$. But given $\epsilon>0$, using $m$, one can find a positive number $A$ depending only on $\eta$, (in particular, independent from $d$ and from the former choice of $\alpha$ ) such that

$$
\begin{gathered}
\left|\int_{-\infty}^{\infty} \frac{(f . \nu)\left(x_{0}\right)}{\left(1+t^{2} \eta(a, \lambda t)\right)^{\frac{3}{2}}} d t-\int_{-A}^{A} \frac{(f . \nu)\left(x_{0}\right)}{\left(1+t^{2} \eta(a, \lambda t)\right)^{\frac{3}{2}}} d t\right|<\epsilon, \\
\left|\int_{-\infty}^{\infty} \frac{(f . \nu)\left(x_{0}\right)}{\left(1+t^{2}\right)^{\frac{3}{2}}} d t-\int_{-A}^{A} \frac{(f . \nu)\left(x_{0}\right)}{\left(1+t^{2}\right)^{\frac{3}{2}}} d t\right|<\epsilon,
\end{gathered}
$$

for all $\lambda$. Then given $A$, one can find $\lambda_{2} \in\left(0, \lambda_{1}\right)$ and $\tilde{\alpha}$ small enough such that

$$
|\eta(a, \lambda t)-1|<\frac{\epsilon}{2 A} \text { for } t \in\left(-\frac{\tilde{\alpha}}{\lambda}, \frac{\tilde{\alpha}}{\lambda}\right) \text { and } \lambda<\lambda_{2} .
$$

Then, using (9.18-9.20) and (9.21), we get

$$
\left|\int_{-\infty}^{\infty} \frac{(f . \nu)\left(x_{0}\right)}{\left(1+t^{2} \eta(a, \lambda t)\right)^{\frac{3}{2}}} d t-\int_{-\infty}^{\infty} \frac{(f . \nu)\left(x_{0}\right)}{\left(1+t^{2}\right)^{\frac{3}{2}}} d t\right|<3 \epsilon .
$$

It is easy to compute that

$$
\int_{-\infty}^{\infty} \frac{1}{\left(1+t^{2}\right)^{\frac{3}{2}}} d t=2
$$

As the choice of $A$ depends only on $\eta$, so does the one of $\tilde{\alpha}$, and we can have chosen in (9.12) the constant $\alpha$ so that $\alpha<\tilde{\alpha}$.

So finally, we have proved that

$$
\int_{\Gamma} \nabla \Theta_{\lambda} \cdot f d \sigma \longrightarrow 2 f\left(x_{0}\right) \cdot \nu\left(x_{0}\right), \text { as } \lambda \rightarrow 0,
$$

that is, exactly (9.9). 
We go back to the proof that $M$ is a tangent measure on $\Gamma$.

We argue by contradiction and suppose that $M . \nu(x) \neq 0$ on the $\operatorname{arc} \gamma\left(\left[x_{0}-\alpha, x_{0}+\alpha\right]\right)$, for a certain $\alpha$ small to be determined. We still denote by $\Gamma_{\alpha}$ the arc $\gamma\left(\left[x_{0}-\alpha, x_{0}+\alpha\right]\right)$.

We can suppose without loss of generality that $\|M . \nu\|_{\mathcal{M}\left(\Gamma_{\alpha}\right)}=1$.

Then given $\epsilon>0$, there exists $f \in C^{0}\left(\Gamma_{\alpha}\right)$ such that

$$
\begin{array}{r}
\|f-M . \nu\|_{\mathcal{M}\left(\Gamma_{\alpha}\right)}<\epsilon, \\
\|f\|_{\mathcal{M}\left(\Gamma_{\alpha}\right)}=1 .
\end{array}
$$

From (9.8) and (9.24), we get that

$$
\left|\int_{\Gamma} f(x) . \nabla \psi d \tau\right|<\epsilon\|\nabla \psi\|_{C^{0}(\Gamma)}
$$

for any $\psi$ satisfying (9.2) and (9.3).

Let us describe the form of functions $\psi$ which will allow us to conclude. Given $\nu(x)$, we consider the curve $\tilde{\gamma}$ obtained by associating to $x$ the point situated at a distance $\lambda$ in the direction $-\nu(x)$ (a kind of "wave front") such as described in Figure 10. We consider also as a parametrisation of $\tilde{\gamma}$ the arc length $\int \mathrm{w}$ ith origin at a certain $\tilde{x}_{0}:=x_{0}-\lambda \nu\left(x_{0}\right)$. As previously, the image of $\tilde{\gamma}$ is denoted by $\tilde{\Gamma}$.

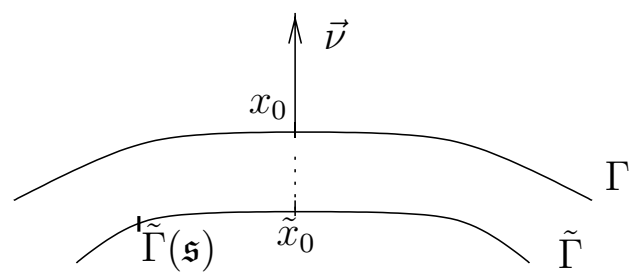

FIgURE 10. The curve $\tilde{\Gamma}$ which supports $\Delta \psi$.

We then consider any function $\mathcal{R}$ of $\mathcal{D}([-\alpha, \alpha])$, with $\alpha$ small to be determined, and define $\psi$ as the integral

$$
\psi(x):=\int_{\mathfrak{s} \in[-\alpha, \alpha]} \mathcal{R}(\mathfrak{s}) \lambda \phi^{\tilde{\gamma}(\mathfrak{s})}(x) d \mathfrak{s} .
$$

Note that $\psi$ satisfies (9.8). Let us prove that $\psi$ is bounded on $\Gamma$ as $\lambda \rightarrow 0$.

For $x_{0}$ in $\Gamma$, we have for a certain $m$ which can be found independent (locally at least) from $x_{0}$, for all $x \in \Gamma$

$$
\int_{\tilde{\Gamma}} \frac{\lambda d y}{|y-x|^{2}} \leq \int_{-s_{0}}^{L-s_{0}} \lambda \frac{d \mathfrak{s}}{\lambda^{2}+m \mathfrak{s}^{2}}, \leq \int_{-\infty}^{+\infty} \frac{d \mathfrak{x}}{1+m \mathfrak{x}^{2}} \leq C(\gamma)
$$

But as we noticed for (9.15), as $x_{0} \notin \partial \Omega$, if we take $\lambda$ small enough (say, inferior to a certain $\lambda_{3}$ ), then one has

$$
\left|\int_{\tilde{\Gamma}}\right| \lambda \nabla \phi^{y}(x)\left|d y-\int_{\tilde{\Gamma}} \frac{\lambda d y}{|y-x|^{2}}\right|<\epsilon
$$

So actually we get that

$$
|\nabla \psi(x)| \leq C(\gamma)\|\mathcal{R}\|_{C_{0}(\Gamma)},
$$


for $x$ near $x_{0}$, as $\lambda \rightarrow 0$.

Considering (9.26) and (9.29), we get that, at least if $\mathcal{R}$ has a small enough support,

$$
\left|\langle f, \nabla \psi\rangle_{\mathcal{M}(\Gamma) \times C^{0}(\Gamma)}\right|<C(\gamma)\|\mathcal{R}\|_{C_{0}(\Gamma)} \epsilon .
$$

With $(9.9,9.29,9.30)$ and the Lebesgue convergence theorem, we get that

$$
\langle f . \nu, \mathcal{R}\rangle_{\mathcal{M}(\Gamma) \times C^{0}(\Gamma)} \leq C(\gamma)\|\mathcal{R}\|_{C_{0}(\Gamma)} \epsilon .
$$

Consequently, with (9.24) we get that

$$
\left|\langle(M . \nu), \mathcal{R}\rangle_{\mathcal{M}(\Gamma) \times C^{0}(\Gamma)}\right|<(1+C(\gamma))\|\mathcal{R}\|_{C_{0}(\Gamma)} \epsilon .
$$

This implies $\|M . \nu\|_{\mathcal{M}(\Gamma)}<C^{\prime}(\gamma) \epsilon$ for every $\epsilon>0$. So we must have $M_{\mid \Gamma((0, L))} . \nu=0$.

Possibly, one could have $M . \nu=\rho_{0} \delta_{\gamma(0)} \cdot \nu+\rho_{1} \delta_{\gamma(L)} \cdot \nu$. Let us explain why necessarily, $\rho_{0}=\rho_{1}=0$. This is a simple consequence of the proof of Lemma 6.2. Indeed, considering as a " $\theta$ " in (9.8) the function $|a-\gamma(0)|^{2} \phi^{a, \bar{a}}$ and $a \rightarrow \gamma(0)$, one could get as a limit value at the point $\gamma(0)$ any tangent vector.

As $\Gamma$ is transverse to $\partial \Omega$ at the point $\gamma(0)$, this implies that $\rho_{0}=0$. The same can be done for $\gamma(L)$.

Since the previous proof is valid whatever the choice of $\nu$, we deduce that $M$ is tangent everywhere on $\Gamma$.

As the second step of the proof of Lemma 9.1, we now check that actually $M=0$. First, we prove $M_{\mid \gamma((0, L))}=$ 0 . As in the previous step, we suppose $M_{\mid \gamma\left(\left[x_{0}-\alpha, x_{0}+\alpha\right]\right)} \neq 0$.

We introduce a function $f \in C^{1}(\Gamma)$ with compact support in $\Gamma_{\alpha}$ so that

$$
\|f-M\|_{\mathcal{M}\left(\Gamma_{\alpha}\right)}<\tilde{\epsilon}
$$

and

$$
\|f\|_{\mathcal{M}\left(\Gamma_{\alpha}\right)} \geq \frac{1}{2}
$$

and moreover that

$$
f \text { is tangent everywhere on } \Gamma \text {. }
$$

In the rest of the proof, we will make no difference between the vectorial function $f$ and the scalar function, which multiplication by the unit tangent vector along $\Gamma$ is $f$.

We now want to prove that $\|f\|_{\mathcal{M}(\Gamma)}<C^{\prime}(\gamma) \tilde{\epsilon}$, for some constant $C^{\prime}(\gamma)$. We proceed as previously, and prove that

$$
\frac{-1}{\log (\lambda)} \int_{\Gamma}\left(\partial_{\tau} f(x)\right) \phi^{a(\lambda)} d \tau \longrightarrow \partial_{\tau} f\left(x_{0}\right), \text { as } \lambda \rightarrow 0
$$

where $a(\lambda)$ is defined (as in the first step) in accordance with Figure 9. As previously, we will use the same arc length $s$ as a parameter on $\Gamma$. Let $\epsilon$ be a positive number. One can find $\alpha>0$, such that

$$
\left|\partial_{\tau} f(x)-\partial_{\tau} f\left(x_{0}\right)\right|<\epsilon \text { on } \Gamma_{\alpha} .
$$

Reducing $\alpha$ if necessary, we demand that for all $s \in(-\alpha, \alpha)$ and for all $\lambda$ less than some $\lambda_{0}$, one has

$$
|\eta(s, \lambda)-\eta(0, \lambda)|<\epsilon
$$


Let us denote by $\tilde{\Theta}^{\lambda}$ the function $-\frac{\phi^{a(\lambda)}}{\log \lambda}$. As for (9.14), one can write

$$
\int_{\Gamma}\left(\partial_{\tau} f(x)\right) \tilde{\Theta}^{\lambda}=\int_{\Gamma_{\alpha}}\left(\partial_{\tau} f(x)\right) \tilde{\Theta}^{\lambda}+\int_{\Gamma \backslash \Gamma_{\alpha}}\left(\partial_{\tau} f(x)\right) \tilde{\Theta}^{\lambda}
$$

For that $\alpha$, one can find $\lambda_{1} \in\left(0, \lambda_{0}\right]$ such that

$$
\left|\int_{\Gamma \backslash \Gamma_{\alpha}}\left(\partial_{\tau} f(x)\right) \tilde{\Theta}^{\lambda} d \tau\right|<\epsilon,
$$

for all $\lambda<\lambda_{1}$. The existence of $\lambda_{1}$ is a simple consequence of the uniform convergence of the function $\phi^{a}$ to 0 on $\Gamma \backslash \Gamma_{\alpha}$ as $a \rightarrow x_{0}$.

From now, we will impose $\lambda$ to be strictly inferior to $\lambda_{0}, \lambda_{1}$ and 1 . Let us then consider the integral of $\tilde{\Theta}^{\lambda} \partial_{\tau} f$ along $\Gamma_{\alpha}$, which we will denote by $\tilde{I}_{1}$. This integral can be estimated the following way :

$$
\begin{aligned}
\tilde{I}_{1} & =\frac{-1}{\log |\lambda|} \int_{-\alpha}^{\alpha} \frac{\partial_{\tau} f(x(s))}{\sqrt{\lambda^{2}+s^{2} \eta(s, \lambda)}} d s \\
& =\frac{-1}{\log |\lambda|} \int_{-\frac{\alpha}{\lambda}}^{\frac{\alpha}{\lambda}} \frac{\left(\partial_{\tau} f\right)(x((t / \lambda))}{\sqrt{1+t^{2} \eta(\lambda t, \lambda)}} d t .
\end{aligned}
$$

We now want to find the limit of this integral. Note that

$$
\left|\frac{-1}{\log |\lambda|} \int_{-\frac{\alpha}{\lambda}}^{\frac{\alpha}{\lambda}} \frac{\partial_{\tau} f(x((t / \lambda))}{\sqrt{1+t^{2} \eta(\lambda t, \lambda)}} d t-\frac{-1}{\log |\lambda|} \int_{-\frac{\alpha}{\lambda}}^{\frac{\alpha}{\lambda}} \frac{\partial_{\tau} f\left(x_{0}\right)}{\sqrt{1+t^{2} \eta(\lambda t, \lambda)}} d t\right| \leq \epsilon\left(2 \lim _{\lambda \rightarrow 0} \frac{-1}{\log |\lambda|} \int_{-\frac{\alpha}{\lambda}}^{\frac{\alpha}{\lambda}} \frac{1}{\sqrt{1+t^{2} m}} d t\right),
$$

if $\lambda_{1}$ is small enough. As

$$
\lim _{\lambda \rightarrow 0} \frac{-1}{\log |\lambda|} \int_{-\frac{K}{\lambda}}^{\frac{K}{\lambda}} \frac{1}{\sqrt{1+t^{2} m}} d t=\frac{1}{\sqrt{m}}
$$

we can deduce the estimate

$$
\left|\frac{-1}{\log |\lambda|}\left(\int_{-\frac{\alpha}{\lambda}}^{\frac{\alpha}{\lambda}} \frac{\partial_{\tau} f(x((t / \lambda))}{\sqrt{1+t^{2} \eta(\lambda t, \lambda)}} d t-\int_{-\frac{\alpha}{\lambda}}^{\frac{\alpha}{\lambda}} \frac{\partial_{\tau} f\left(x_{0}\right)}{\sqrt{1+t^{2} \eta(\lambda t, \lambda)}} d t\right)\right| \leq 2 \epsilon \sqrt{m}^{-1} .
$$

Let us define

$$
\tilde{I}_{2}:=-\frac{1}{|\lambda|} \int_{-\frac{\alpha}{\lambda}}^{\frac{\alpha}{\lambda}} \frac{\partial_{\tau} f\left(x_{0}\right)}{\sqrt{1+t^{2} \eta(\lambda t, \lambda)}} d t
$$

Using (9.38), we can deduce:

$$
\frac{-1}{\log |\lambda|} \int_{-\frac{\alpha}{\lambda}}^{\frac{\alpha}{\lambda}} \frac{\partial_{\tau} f\left(x_{0}\right)}{\sqrt{1+(1+\epsilon) t^{2}}} d t \leq I_{2} \leq \frac{-1}{\log |\lambda|} \int_{-\frac{\alpha}{\lambda}}^{\frac{\alpha}{\lambda}} \frac{\partial_{\tau} f\left(x_{0}\right)}{\sqrt{1+(1-\epsilon) t^{2}}} d t
$$

if $\partial_{\tau} f\left(x_{0}\right)>0$ and in the other way around otherwise. Consequently,

$$
(1-\epsilon) \lim _{\lambda \rightarrow 0} \frac{-1}{\log |\lambda|} \int_{-\frac{\alpha}{\lambda}}^{\frac{\alpha}{\lambda}} \frac{\partial_{\tau} f\left(x_{0}\right) d t}{\sqrt{1+(1+\epsilon) t^{2}}} \leq I_{2}
$$


and

$$
I_{2} \leq(1+\epsilon) \lim _{\lambda \rightarrow 0} \frac{-1}{\log |\lambda|} \int_{-\frac{\alpha}{\lambda}}^{\frac{\alpha}{\lambda}} \frac{\partial_{\tau} f\left(x_{0}\right) d t}{\sqrt{1+(1-\epsilon) t^{2}}}
$$

which implies

$$
(1-3 \epsilon) \partial_{\tau} f\left(x_{0}\right) \leq \frac{-1}{\log |\lambda|} \int_{-\frac{\alpha}{\lambda}}^{\frac{\alpha}{\lambda}} \frac{\partial_{\tau} f\left(x_{0}\right)}{\sqrt{1+t^{2} \eta(\lambda t, \lambda)}} d t \leq(1+3 \epsilon) \partial_{\tau} f\left(x_{0}\right) .
$$

From $(9.40,9.43)$ and $(9.45)$ we get that for $\lambda<\lambda_{1}$,

$$
\left|\int_{\Gamma} \tilde{\Theta}(x) \partial_{\tau} f(x) d x-\partial_{\tau} f\left(x_{0}\right)\right|<\left(1+\left(3+2 \sqrt{m}^{-1}\right)\left\|\partial_{\tau} f\right\|_{C^{0}}\right) \epsilon .
$$

So we have obtained (9.36).

We go back to the proof that $M=0$. We integrate by parts to obtain the general equality

$$
\int_{\Gamma} f . \nabla \psi=f(\gamma(0)) \psi(\gamma(0))-f(\gamma(L)) \psi(\gamma(L))+\int_{\Gamma} \psi \partial_{\tau} f d \tau .
$$

Considering $|a-\gamma(0)| \phi^{a}$ as a function $\psi$, and making $a$ converge to $\gamma(0)$, we obtain easily that

$$
|f(\gamma(0))|<\epsilon,
$$

and the same for $\gamma(L)$ (indeed this family of functions is uniformly bounded by 1 and converges to the characteristic function of $\gamma(0)$ when $a \rightarrow \gamma(0))$.

After that we consider a function $\psi$ defined exactly as in the previous step, with a support described by Figure 10. Again we introduce a smooth function $\mathcal{R}$ with support $[-\alpha, \alpha](\alpha$ small). Then we define the function $\psi$ by

$$
\psi(x):=\int_{\mathfrak{s} \in[-\alpha, \alpha]} \mathcal{R}(\mathfrak{s}) \frac{-1}{\log \lambda} \phi^{\tilde{\gamma}(\mathfrak{s})}(x) d \mathfrak{s} .
$$

We can prove that $\psi$ is bounded when $d \rightarrow 0$, in the same way as is the first step.

We get then by $(9.26,9.36,9.48)$ and $(9.49)$, using the same deduction as in the previous step, that

$$
\|f\|_{\mathcal{M}(\Gamma)}<C^{\prime}(\gamma) \epsilon
$$

which is a contradiction with (9.34) for $\epsilon$ small enough.

\subsection{Proof of Corollary 9.2}

Before precisely proving Corollary 9.2, we state the following intermediate result:

Corollary 9.3. Given $\gamma$ as in Lemma 9.1, for any $\epsilon>0$, there exists some positive constant $W(\gamma, \epsilon)$ such that for any $y$ satisfying the assumptions of Lemma 9.1, which is moreover in $C^{1}\left(\Gamma ; \mathbb{R}^{3}\right)$, there exists a function $\theta$ satisfying (9.2-9.3) such that

$$
\|y-\nabla \theta\|_{C^{0}\left(\Gamma ; \mathbb{R}^{3}\right)}<\epsilon\|y\|_{C^{0}\left(\Gamma ; \mathbb{R}^{3}\right)}
$$


and furthermore the inequality

$$
\|\theta\|_{C^{2}(\bar{\Omega})} \leq W(\gamma, \epsilon)\|y\|_{C^{1}\left(\Gamma ; \mathbb{R}^{3}\right)}
$$

Let us first remark that, if we do not take (9.51) into account, there is no difference between Corollary 9.3 and Lemma 9.1.

To prove this corollary, we argue by contradiction, and suppose that there exists a certain $\epsilon_{0}>0$, such that for any $n \in \mathbb{N}$, one can find $y_{n} \in C^{1}\left(\Gamma ; \mathbb{R}^{3}\right)$, such that for any $\theta$ satisfying (9.2-9.3) and (9.50) with $y=y^{n}$ and $\epsilon=\epsilon_{0}$, then one has

$$
\|\theta\|_{C^{2}(\bar{\Omega})} \geq n\left\|y^{n}\right\|_{C^{1}\left(\Gamma ; \mathbb{R}^{3}\right)} .
$$

We can obviously suppose that $\left\|y^{n}\right\|_{C^{1}\left(\Gamma ; \mathbb{R}^{3}\right)}=1$, by "homogeneity" of formula (9.50).

By Ascoli's theorem, we can suppose that

$$
y^{n} \stackrel{C^{0}\left(\Gamma ; \mathbb{R}^{3}\right)}{\longrightarrow} Y, \text { as } n \rightarrow \infty .
$$

We use Lemma 9.1 with $y=Y$ and $\epsilon=\frac{1}{2} \epsilon_{0}$, and hence we get a certain $\bar{\theta}$. Then, by (9.53), for $n$ large enough, one has $\left\|\nabla \bar{\theta}-y^{n}\right\|_{C^{0}\left(\Gamma ; \mathbb{R}^{3}\right)}<\epsilon_{0}\left\|y^{n}\right\|_{C^{0}\left(\Gamma ; \mathbb{R}^{3}\right)}$.

By (9.52), we deduce

$$
\|\bar{\theta}\|_{C^{2}(\bar{\Omega})} \geq n
$$

for all $n \in \mathbb{N}$, which is obviously absurd.

Our goal is now, in order to prove Corollary 9.2, to check that one can find in the constant $W(\gamma, \epsilon)$ independently from $\gamma$ (at least for curves "close" to $\gamma$ ).

Let us indeed consider near $\gamma$ a different arc $\tilde{\gamma}$ such that $\|\gamma-\tilde{\gamma}\|_{C^{0}}<\beta$ for a certain $\beta>0$. We consider a certain $\tilde{y}$ in $C^{1}\left(\tilde{\Gamma} ; \mathbb{R}^{3}\right)$ which satisfies the same assumption as $y$ in Lemma 9.1. Then one can extend $\tilde{y}$ to a $C^{1}$ vector field in $\bar{\Omega}$, in such a way that $\|y\|_{C^{1}\left(\bar{\Omega} ; \mathbb{R}^{3}\right)}=\|\tilde{y}\|_{C^{1}\left(\tilde{\Gamma} ; \mathbb{R}^{3}\right)}$.

Now we consider the $\theta$ given by Corollary 9.3 on $\gamma$ with a given $\epsilon$, and the function $y_{\mid \gamma}$.

We want to show this $\theta$ solves a similar problem on $\tilde{\gamma}$. For any $s \in[0, L]$, one can compute

$$
|\nabla \theta(\tilde{\gamma}(s))-\tilde{y}(\tilde{\gamma}(s))| \leq|\nabla \theta(\tilde{\gamma}(s))-\nabla \theta(\gamma(s))|+|\nabla \theta(\gamma(s))-y(\gamma(s))|+|y(\gamma(s))-\tilde{y}(\tilde{\gamma}(s))| .
$$

Using the second derivatives of $\theta$, one can deduce

$$
|\nabla \theta(\gamma(s))-\nabla \theta(\tilde{\gamma}(s))|<W(\epsilon) \beta\|y\|_{C^{1}\left(\tilde{\Gamma} ; \mathbb{R}^{3}\right)} .
$$

By the choice of $y$, we have

$$
|y(\gamma(s))-\tilde{y}(\tilde{\gamma}(s))|<\|\tilde{y}\|_{C^{1}\left(\tilde{\Gamma} ; \mathbb{R}^{3}\right)} \beta .
$$

And $\theta$ was chosen such that

$$
|\nabla \theta(\gamma(s))-y(\gamma(s))|<\epsilon\|y\|_{C^{0}\left(\Gamma ; \mathbb{R}^{3}\right)}
$$

Consequently, we have finally

$$
|\nabla \theta(\tilde{\gamma}(s))-\tilde{y}(\tilde{\gamma}(s))|<(W(\epsilon) \beta+2 \beta)\|\tilde{y}\|_{C^{1}\left(\tilde{\Gamma} ; \mathbb{R}^{3}\right)}+\epsilon\|y\|_{C^{0}\left(\Gamma ; \mathbb{R}^{3}\right)} .
$$


Taking $\beta:=\frac{\epsilon}{W(\epsilon)+2}$, we obtain Corollary 9.2.

\subsection{Proof of Lemma 7.1}

Before defining $\overline{\mathbf{v}}:=\nabla \theta$, we would like to make a small transformation on $\mathbf{v}$, which one can perform for any $q \in \mathbb{N}^{*}$ and any $\tau<\frac{1}{3 q}$.

We introduce a function $p: \mathbb{R} \rightarrow \mathbb{R}$, such that

$$
\begin{gathered}
p=1 \text { in }\left(-\infty,-\frac{3}{2}\right] \cup[0,+\infty), \\
p=0 \text { in }\left[-1,-\frac{1}{2}\right] \\
\|p\|_{C^{0}} \leq 1, \quad\|p\|_{C^{1}} \leq 10 .
\end{gathered}
$$

We also introduce a function $j$ such that

$$
\begin{gathered}
j=0 \text { on }(-\infty, 0] \cup[1 / 2,+\infty), \\
0 \leq j \leq 10 \text { in } \mathbb{R}, \\
\int_{\mathbb{R}} j=1 .
\end{gathered}
$$

We consider times $t_{i}:=\frac{i}{q}$, for $i \in\{0, \ldots, q\}$. Then we define $\hat{\mathbf{v}}_{\tau, q}$ on the time interval $\left[t_{i}, t_{i+1}\right], i \in\{0, \ldots, q-1\}$ by

$$
\hat{\mathbf{v}}_{\tau, q}(t, x)=p\left(\frac{t-t_{i+1}}{\tau}\right) \mathbf{v}(t, x) \text { in }\left[t_{i}, t_{i+1}\right] \times \bar{\Omega}
$$

with $\tau$ to be chosen small enough. Note in particular that

$$
\|\hat{\mathbf{v}}\|_{C^{0}\left([0,1], C^{1}(\bar{\Omega})\right)} \leq\|\mathbf{v}\|_{C^{0}\left([0,1], C^{1}(\bar{\Omega})\right)} .
$$

Let us prove that, given $q$, one has $\left|\phi^{\mathbf{v}}(0, t, x)-\phi^{\hat{\mathbf{v}}_{\tau, q}}(0, t, x)\right|$ uniformly small if $\tau$ is small. This is done by an induction method.

We consider the evolution of both flows of $\mathbf{v}$ and $\hat{\mathbf{v}}_{\tau, q}$ between timest $t_{i}$ and $t_{i+1}-\frac{3 \tau}{2}$. For these times, one has $\hat{\mathbf{v}}_{\tau, q}=\mathbf{v}$, so by Gronwall's lemma we obtain the inequality for all $t \in\left[t_{i}, t_{i+1}-\frac{3 \tau}{2}\right]$

$$
\left|\phi^{\mathbf{v}}(x, 0, t)-\phi^{\hat{\mathbf{v}}_{\tau, q}}(x, 0, t)\right| \leq\left|\phi^{\mathbf{v}}\left(0, t_{i}, x\right)-\phi^{\hat{\mathbf{v}}_{\tau, q}}\left(0, t_{i}, x\right)\right| t e^{t\|\mathbf{v}\|_{C^{0}}}
$$

But is is quite clear that for all $t$ in $\left[t_{i+1}-\frac{3 \tau}{2}, t_{i+1}\right]$, one has

$$
\left|\phi^{\mathbf{v}}(x, 0, t)-\phi^{\hat{\mathbf{v}}_{\tau, q}}(x, 0, t)\right| \leq\left|\phi^{\mathbf{v}}\left(0, t_{i+1}-\frac{3 \tau}{2}, x\right)-\phi^{\hat{\mathbf{v}}_{\tau, q}}\left(0, t_{i+1}-\frac{3 \tau}{2}, x\right)\right|+2 \tau\|\mathbf{v}\|_{C^{0}\left([0,1], C^{1}(\bar{\Omega})\right)}
$$


If we consider the sequence $\left(u_{n}\right)$ defined by

$$
\left\{\begin{array}{l}
u_{0}=0, \\
u_{n+1}=u_{n} e^{\frac{\|\mathbf{v}\|_{C^{0}\left([0,1], C^{1}(\bar{\Omega})\right)}^{q}}{q}}+2 \tau\|\mathbf{v}\|_{C^{0}\left([0,1], C^{1}(\bar{\Omega})\right)},
\end{array}\right.
$$

then we get

$$
\left|\phi^{\mathbf{v}}(x, 0, t)-\phi^{\hat{\mathbf{v}}_{\tau, q}}(x, 0, t)\right| \leq u_{q},
$$

for all $t \in[0,1]$.

Let

$$
b:=e^{\frac{\|\mathbf{v}\|_{C^{0}\left([0,1], C^{1}(\bar{\Omega})\right)}^{q}}{q}} \text { and } c:=2\|\mathbf{v}\|_{C^{0}\left([0,1], C^{1}(\bar{\Omega})\right)} \tau .
$$

We have

$$
u_{n}=\frac{c}{1-b}+\left(\frac{c}{b-1}\right) b^{n}
$$

As $b^{q}=e^{\|\mathbf{v}\|_{C^{0}\left([0,1], C^{1}(\bar{\Omega})\right)}}$, we obtain that $u_{q}$ is small when $\tau$ is small, that is what we intended to prove. Precisely, if one expects

$$
\left|\phi^{\mathbf{v}}(x, 0, t)-\phi^{\hat{\mathbf{v}}_{\tau, q}}(x, 0, t)\right|<\frac{\epsilon}{2},
$$

then it is sufficient to take as $\tau$ the real number (if $k$ is large)

$$
\tau(\epsilon, k):=\frac{\epsilon}{4 k} .
$$

We go back to the problem of approximation of $\mathbf{v}$ by $\mathbf{v}$. Let us denote by $J(t)$ the $\operatorname{arc} \phi^{\mathbf{v}}(J, 0, t)$. It is parametrized by $s \in[0,1]$. We consider $\epsilon \in(0,1 / 400]$. For every $t \in[0,1]$, one can deduce by Corollary 9.2 a certain $\delta(J(t), \epsilon / 2)$ and a certain $\mathcal{W}(J(t), \epsilon / 2)$.

Let us remark that, if $\tilde{t}$ is close enough to $t$, then one can expect the relation $\|J(t)-J(\tilde{t})\|_{C^{0}\left([0,1], C^{1}(\bar{\Omega})\right)}<$ $\delta(\epsilon / 2, J(t)) / 2$.

So for fixed $t$, if we increase $\mathcal{W}$ and decrease $\delta$, we can consider them valid for the $\operatorname{arcs} \gamma(\tilde{t})$, with $\tilde{t}$ in a small open set around $t$. Then by compacity of the interval [0,1], one can find $\delta(\epsilon)$ and $\mathcal{W}(\epsilon)$ valid for all $\gamma$ in the set $\{J(t), t \in[0,1]\}$. We furthermore impose

$$
\delta<\epsilon / 20 \text { and } \delta \mathcal{W}<\epsilon / 2,
$$

reducing $\delta$ if needed.

Then, we fix $k$ in $\mathbb{N}$ such that

$$
\left(20 \epsilon \delta+\frac{2 \epsilon\|\mathbf{v}\|_{C^{0}\left([0,1], C^{1}(\bar{\Omega})\right)}}{k}\right) e^{\frac{\mathcal{W}\|\mathbf{v}\|}{C^{0}\left([0,1], C^{1}(\bar{\Omega})\right)}} k<\frac{\delta}{k} .
$$

We also require from $k$ that for any $t, t^{\prime} \in[0,1]$ such that $\left|t-t^{\prime}\right|<\frac{1}{k}$, one has

$$
\left|\mathbf{v}(t, \cdot)-\mathbf{v}\left(t^{\prime}, \cdot\right)\right|_{C^{0}(\bar{\Omega})}<\epsilon\|\mathbf{v}\|_{C^{0}},
$$


which can be obtained by an argument of uniform continuity.

We cut the time interval $[0,1]$ in $k$ parts $\left[\frac{l}{k}, \frac{l+1}{k}\right]$, with $l=0, \ldots, k-1$. From now we denote by $t_{l}$ the time $l / k$. For that $k$, we introduce a $\tau$ such that $\left|\left(\phi^{\hat{\mathbf{v}}_{k, \tau}}-\phi^{\mathbf{v}}\right)(x, 0, t)\right|<\epsilon$ for any $(t, x) \in[0,1] \times \bar{\Omega}(\operatorname{expressed}$ by (9.73)). From now, we will consider $\hat{\mathbf{v}}_{k, \tau}$ instead of $\mathbf{v}$ and particularly we will set $\hat{J}(t):=\phi^{\hat{\mathbf{v}}_{k, \tau}}\left(J_{0}, 0, t\right)$.

We will define $\overline{\mathbf{v}}$ inductively on these time intervals, so that it will be close to $\hat{\mathbf{v}}_{k, \tau}$.

We will denote by $\mathcal{T}(\gamma, y, \epsilon)$ a function $\theta$ given by Corollary 9.2 with $\gamma, y$ and $\epsilon$ as variables. At each time, we introduce the arc

$$
\mathcal{J}(t):=\phi^{\overline{\mathbf{v}}}\left(J_{0}, 0, t\right)
$$

and we will denote by $G(s, t)$ the function defined on $[0,1] \times[0,1]$ by

$$
G(\cdot, t)=\frac{\hat{J}(t)-\mathcal{J}(t)}{\tau}
$$

Both functions $\mathcal{J}$ and $G$ are well defined along the construction of $\overline{\mathbf{v}}$.

During the time interval $\left[0, \frac{1}{k}-\tau\right]$, we set

$$
\overline{\mathbf{v}}(t, x)=p\left(\frac{t-t_{1}}{\tau}\right) \mathcal{T}\left(J_{0}, \mathbf{v}(0, \cdot), \epsilon / 2\right)
$$

Then we define $\overline{\mathbf{v}}$ during the second time interval $\left[\frac{1}{k}-\tau, \frac{1}{k}-\frac{\tau}{2}\right]$ by

$$
\overline{\mathbf{v}}(t, x)=j\left(\frac{t-t_{1}+\tau}{\tau}\right) \mathcal{T}\left(\mathcal{J}\left(t_{1}-\tau\right), G\left(\cdot, t_{1}-\tau\right), \epsilon / 2\right) .
$$

We then define $\overline{\mathbf{v}}$ by induction on the intervals $\left[\frac{l}{k}-\frac{\tau}{2}, \frac{l+1}{k}-\tau\right]$ by

$$
\overline{\mathbf{v}}(t, x)=p\left(\frac{t-t_{l+1}}{\tau}\right) \mathcal{T}\left(\mathcal{J}\left(t_{l}\right), \mathbf{v}\left(t_{l}, \cdot\right), \epsilon / 2\right),
$$

and during the intervals: $\left[\frac{l+1}{k}-\tau, \frac{l+1}{k}-\frac{\tau}{2}\right]$, we set

$$
\overline{\mathbf{v}}(t, x)=j\left(\frac{t-t_{l}+\tau}{\tau}\right) \mathcal{T}\left(\mathcal{J}\left(t_{l}-\tau\right), G\left(\cdot, t_{l}-\tau\right), \epsilon / 2\right)
$$

When $k=l-1$, we extend this formula till $t=1$.

Note that this can be done, because the assumptions on $\gamma$ (injectivity in particular) in Corollary 9.2 are preserved by the flow of $\mathbf{v}$.

Now we have to prove that the $\overline{\mathbf{v}}$ constructed above has the required properties. We want to estimate the norm $\left\|\phi^{\overline{\mathbf{v}}}\left(J_{0}, 0, t\right)-\phi^{\mathbf{v}}\left(J_{0}, 0, t\right)\right\|_{C^{0}([0,1])}$ (as the constants $k$ and $\tau$ are now fixed, we will no longer precise them for $\hat{\mathbf{v}}$ ).

We compute

$$
\frac{d}{d t^{+}}|\mathcal{J}(t)(s)-\hat{J}(t)(s)| \leq|\overline{\mathbf{v}}(\mathcal{J}(t)(s))-\hat{\mathbf{v}}(\hat{J}(t)(s))| .
$$

We want to prove that for all $t \in[0,1]$, one has

$$
|G(\cdot, t)|<\frac{\delta}{\tau}
$$


and

$$
\left|G\left(\cdot, t_{l}-\frac{\tau}{2}\right)\right|<\frac{20 \epsilon \delta}{\tau} .
$$

We prove jointly that (9.84) is true on the intervals $\left[t_{l}-\frac{\tau}{2}, t_{l+1}-\frac{\tau}{2}\right]$ and (9.85) by induction on $l$.

For $l=0$, as we have chosen the time support of $\mathbf{v}$ placed after $t_{1}$, we have nothing to prove.

Let us suppose (9.84) true on the interval $\left[t_{l-1}-\frac{\tau}{2}, t_{l}-\frac{\tau}{2}\right]$ and also (9.85) at rank $l$. Then, we first consider what happens during the time interval $\left[t_{l}-\frac{\tau}{2}, t_{l+1}-\tau\right]$. By (9.83), one has

$$
\begin{aligned}
\frac{d}{d t^{+}}|\mathcal{J}(t)(s)-\hat{J}(t)(s)| & \leq\left|\overline{\mathbf{v}}(\mathcal{J}(t)(s))-\overline{\mathbf{v}}\left(\hat{J}\left(t_{l}-\frac{\tau}{2}\right)(s)\right)\right|+\left|\overline{\mathbf{v}}\left(\hat{J}\left(t_{l}-\frac{\tau}{2}\right)(s)\right)-\hat{\mathbf{v}}\left(\hat{J}\left(t_{l}-\frac{\tau}{2}\right)(s)\right)\right| \\
& +\left|\hat{\mathbf{v}}\left(\hat{J}\left(t_{l}-\frac{\tau}{2}\right)(s)\right)-\hat{\mathbf{v}}(\hat{J}(t)(s))\right| .
\end{aligned}
$$

Consequently, one has

$$
\frac{d}{d t^{+}}|\mathcal{J}(t)(s)-\hat{J}(t)(s)| \leq \mathcal{W}\|\mathbf{v}\||\mathcal{J}(t)(s)-\hat{J}(t)(s)|+\epsilon\|\mathbf{v}\|+\epsilon\|\mathbf{v}\|,
$$

where the norm considered is the norm in $C^{0}\left([0,1], C^{1}(\bar{\Omega})\right)$. This gives, when integrated, for all $t \in\left[t_{l}-\frac{\tau}{2}, t_{l+1}-\right.$ $\tau]$,

$$
|\mathcal{J}(t)(s)-\hat{J}(t)(s)| \leq\left|\mathcal{J}\left(t_{l}-\frac{\tau}{2}\right)(s)-\hat{J}\left(t_{l}-\frac{\tau}{2}\right)(s)\right|+\frac{2 \epsilon\|\mathbf{v}\|}{k}+\int_{t_{l}-\frac{\tau}{2}}^{t} \mathcal{W}\|\mathbf{v}\||\mathcal{J}(t)(s)-\hat{J}(t)(s)|,
$$

which implies by the induction hypothesis and Gronwall's lemma

$$
|\mathcal{J}(t)(s)-\hat{J}(t)(s)| \leq\left(20 \epsilon \delta+\frac{2 \epsilon\|\mathbf{v}\|}{k}\right) e^{\mathcal{W}\|\mathbf{v}\| t-t_{l}+\frac{\tau}{2}} .
$$

Together with (9.75), this gives us for $t \in\left[t_{l}-\frac{\tau}{2}, t_{l+1}-\tau\right]$

$$
|\mathcal{J}(t)(s)-\hat{J}(t)(s)|<\frac{\delta}{20}
$$

which implies a fortiori relation (9.84) on the interval $\left[t_{l}-\frac{\tau}{2}, t_{l+1}-\tau\right]$. Let us prove it on $\left[t_{l+1}-\frac{3 \tau}{2}, t_{l+1}-\frac{\tau}{2}\right]$.

We make the same computation as in (9.86) and get for $t$ in $\left[t_{l+1}-\tau, t_{l+1}-\frac{\tau}{2}\right]$

$$
\frac{d}{d t^{+}}|\mathcal{J}(t)(s)-\hat{J}(t)(s)| \leq \mathcal{W}\left\|j(t) G\left(t_{l}-\frac{\tau}{2}\right)\right\||\mathcal{J}(t)(s)-\hat{J}(t)(s)|+\epsilon\|G\|
$$

Therefore

$$
|\mathcal{J}(t)(s)-\hat{J}(t)(s)| \leq \frac{\delta}{20}+\tau \epsilon\left\|j\left(t_{l}-\frac{\tau}{2}\right) G\left(t_{l}-\frac{\tau}{2}\right)\right\|+\int_{t_{l}-\frac{\tau}{2}}^{t} 10 \mathcal{W}\|G\||\mathcal{J}(t)(s)-\hat{J}(t)(s)|,
$$

which implies, by Gronwall's lemma,

$$
\left.|\mathcal{J}(t)(s)-\hat{J}(t)(s)| \leq\left(\frac{\delta}{10}\right) e^{10 \mathcal{W}\|G\|\left(t-t_{l}+\frac{\tau}{2}\right.}\right), \leq \frac{\delta}{10} e^{10 W \delta}, \leq \frac{\delta}{2},
$$


and hence, (9.84) holds on the interval $\left[t_{l+1}-\tau, t_{l+1}-\frac{\tau}{2}\right]$.

Then, in order to prove (9.85) at rank $l+1$, we consider the difference for $t \in\left[t_{l+1}-\tau, t_{l+1}-\frac{\tau}{2}\right]$ :

$$
\left|\phi^{\overline{\mathbf{v}}}\left(t_{l}-\tau, t, \mathcal{J}\left(t_{l}-\tau\right)\right)-\phi^{G(s, t)}\left(t_{l}-\tau, t, \mathcal{J}\left(t_{l}-\tau\right)\right)\right|
$$

But one has

$$
\|\overline{\mathbf{v}}(\mathcal{J}(t)(s), t)-j(t) G(s, t)\| \leq 10 \epsilon\|G\|+10 \mathcal{W}\|G\||\mathcal{J}(t)(s)-\hat{J}(t)(s)|, \leq 10(\epsilon+\mathcal{W} \delta)\|G\|
$$

from what we deduce

$$
\left|\phi^{\overline{\mathbf{v}}}\left(t_{l}-\tau, t, \mathcal{J}\left(t_{l}-\tau\right)\right)-\phi^{j(t) G(s, t)}\left(t_{l}-\tau, t, \mathcal{J}\left(t_{l}-\tau\right)\right)\right| \leq(\epsilon+\mathcal{W} \delta) 10\|G\| \tau, \leq 10 \delta(\epsilon+\mathcal{W} \delta),
$$

which gives us (cf. (9.74)), if we take $t=t_{l+1}-\frac{\tau}{2}$, relation (9.85) at rank $l$.

Note that we could use the estimate of Corollary 9.2 because $\mathcal{J}(t)$ was not too far from $\hat{J}(t)$.

Then (9.84) implies

$$
|\hat{J}(t)-\mathcal{J}(t)|<\epsilon,
$$

for all $t$ in $[0,1]$.

This concludes the proof of the first statement of Lemma 7.1.

\subsection{The second statement of Lemma 7.1}

In this case the demonstration of an equivalent Lemma 9.1 is the same. First, one shows that the measure $M$ is necessarily tangent on $J_{0}$, then that it must be a linear vortex on $J_{0}$ exactly in the same way. But this time the conclusion that $M$ is zero does not come from a particular study of points $\gamma(0)$ and $\gamma(1)$, but from the additional assumption (7.3).

Then, the conclusions given by Corollary 9.2 and Subsection 9.4 are still valid, which completes the proof of Lemma 7.1.

\section{REFERENCES}

[1] C. Bardos and U. Frisch, Finite-time regularity for bounded and unbounded ideal incompressible fluids using Hölder estimates, in Proceedings of the conference held at the university of Paris-Sud Orsay, France. Springer-Verlag, Lectures Notes in Math. $\mathbf{5 6 5}(1975)$ 1-13.

[2] J.-M. Coron, Global Asymptotic Stabilization for controllable systems without drift. Math. Control Signals Systems 5 (1992) 295-312.

[3] J.-M. Coron, Contrôlabilité exacte frontière de l'équation d'Euler des fluides parfaits incompressibles bidimensionnels. $C$. $R$. Acad. Sci. Paris Sér. I Math. 317 (1993) 271-276.

[4] J.-M. Coron, On the controllability of 2-D incompressible perfect fluids. J. Math. Pures Appl. 75 (1996) 155-188.

[5] J.-M. Coron, On the controllability of the 2-D incompressible Navier-Stokes equations with the Navier slip boundary conditions. ESAIM Control Optim. Calc. Var. 1 (1996) 35-75. http://www.emath.fr/cocv/.

[6] O. Glass, Exact boundary controllability of 3-D Euler equation of perfect incompressible fluids. C. R. Acad. Sci. Paris Sér. I Math. 325 (1997) 987-992.

[7] O. Glass, Contrôlabilité de l'équation d'Euler tridimensionnelle pour les fluides parfaits incompressibles, Séminaire Équations aux Dérivées Partielles, 1997-1998, École polytechnique, Centre de Mathématiques, exposé XV.

[8] P. Hermann and H. Kersten, Über die stetige Abhängigkeit der Lösung des Neumann-Problems für die Prae-Maxwellschen Gleichungen von ihren Randdaten. Arch. Math. (Basel) 36 (1981) 79-82.

[9] A.V. Kazhikov, Note on the formulation of the problem of flow through a bounded region using equations of perfect fluid. PMM USSR 44 (1981) 672-674.

[10] J.-L. Lions, Are there connections between turbulence and controllability?, 9th INRIA International Conference, Antibes (June $12-15,1990)$.

[11] R. Temam, Navier-Stokes equations and numerical analysis. North-Holland Pub. (1979). 